TRANSACTIONS OF THE

AMERICAN MATHEMATICAL SOCIETY

Volume 355, Number 5, Pages 1803-1818

S 0002-9947(03)03245-8

Article electronically published on January 14, 2003

\title{
CYCLOTOMIC UNITS AND STICKELBERGER IDEALS OF GLOBAL FUNCTION FIELDS
}

\author{
JAEHYUN AHN, SUNGHAN BAE, AND HWANYUP JUNG
}

\begin{abstract}
In this paper, we define the group of cyclotomic units and Stickelberger ideals in any subfield of the cyclotomic function field. We also calculate the index of the group of cyclotomic units in the total unit group in some special cases and the index of Stickelberger ideals in the integral group ring.
\end{abstract}

\section{INTRODUCTION}

In the cyclotomic number field $\mathbb{Q}\left(\zeta_{n}\right)$, where $\zeta_{n}=\exp (2 \pi i / n)$, Sinnott S1] showed that the index of cyclotomic units in the total unit group is equal to the class number of its maximal real subfield up to a simple constant factor (called the Kummer-Sinnott unit-index formula) and the index of the Stickelberger ideal associated to $\mathbb{Q}\left(\zeta_{n}\right)$ is equal to the relative class number up to a simple constant factor (called the Iwasawa-Sinnott index formula of the Stickelberger ideal). In S2], he also extended these results to arbitrary abelian number fields over $\mathbb{Q}$. The analogue of Kummer-Sinnott's unit-index formula, with the Carlitz module assigned to the role played in the classical cyclotomic theory by the multiplicative group, was carried out by Galovich and Rosen [GR in the rational function field case and by Yin [Y1], replacing the Carlitz module by a general sign-normalized rankone Drinfeld module in the global function field case. Harrop $[\mathrm{Hr}]$ extended the Galovich-Rosen result to any subfield of a cyclotomic function field over the rational function field. The analogue of Iwasawa-Sinnott's index formula of the Stickelberger ideal was carried out by Yin [Y2], which says that the index of the Stickelberger ideal is equal to the relative ideal class number of the field up to a simple constant factor in the global function field case. Recently Yin [Y3 also defined an ideal (he also called it the Stickelberger ideal) in the integral group ring relative to any finite abelian extension of global fields whose rank is equal to the degree of the extension in the function field case. In (wide or narrow) ray class extension of function fields, he calculated the index of the Stickelberger ideal in the integral group ring, which is equal to the divisor class number up to a simple constant factor.

In this article we study the cyclotomic units and the Stickelberger ideal of some abelian extensions of a global function field. Let $k$ be a global function field. Let $F / k$ be a finite abelian extension which is a subfield of a cyclotomic function field. In section 2 , we recall some notation and results of a cyclotomic function field over

Received by the editors July 1, 2001 and, in revised form, October 28, 2002.

2000 Mathematics Subject Classification. Primary 11R58, 11R60.

Key words and phrases. cyclotomic units, Stickelberger ideal, global function field.

This work was supported by Korea Research Foundation Grant (KRF-2000-015-DP0010). 
a global function field and earlier results needed in the paper. In section 3 , we define the group of cyclotomic units $C_{F}$ and then calculate its index in the total unit group $\mathcal{O}_{F}^{*}$ in some special cases (Thm. 3.9), which involves $h\left(\mathcal{O}_{F^{+}}\right)$, the ideal class number of its maximal real subfield $F^{+}$. The group of cyclotomic units in this paper (Def.3.1) is smaller than that in $\mathrm{Y1}$ in the case of cyclotomic extensions. In fact, it is the unramified part that is smaller than Yin's. In section 4 , we define the Stickelberger ideals $I_{F}^{ \pm}$and $I_{F}$ and calculate their indices $\left[R^{ \pm}: I_{F}^{ \pm}\right]$and $\left[R: I_{F}\right]$ whose formulas involve the class numbers $h^{-}\left(\mathcal{O}_{F}\right), h\left(F^{+}\right)$and $h(F)$, respectively (Thms. 4.7 4.11 and 4.12). In section 5, we discuss the indices $(R: U),\left(e^{+} R: e^{+} U\right)$ and $\left(e^{-} R: e^{-} U\right)$ that appear in our index formulas.

\section{Preliminaries}

Let $k$ be a global function field with constant field $\mathbb{F}_{q}$ of $q$ elements, and let $\infty$ be a fixed place of $k$ with degree one. Let $k_{\infty}$ be the completion of $k$ at $\infty$ and $\Omega$ be the completion of an algebraic closure of $k_{\infty}$. Let $\mathbb{A}$ be the Dedekind ring of functions in $k$ that are holomorphic away from $\infty$. We fix a sign function sgn $: k_{\infty} \rightarrow \mathbb{F}_{q}$ with $\operatorname{sgn}(0)=0$ (Def. 4.1, [H2] $)$. An element $z$ of $k_{\infty}^{*}$ is called positive if $\operatorname{sgn}(z)=1$.

For any nonzero integral ideal $\mathfrak{m}$ of $\mathbb{A}$, we denote by $K_{\mathfrak{m}}$ the cyclotomic function field of the triple $(k, \infty, \operatorname{sgn})$ of conductor $\mathfrak{m}$ and by $K_{\mathfrak{m}}^{+}$its maximal real subfield. In particular, if $\mathfrak{m}=\mathfrak{e}$, the unit ideal of $\mathbb{A}$, then $K_{\mathfrak{e}}$ is the Hilbert class field of the triple $(k, \infty, \mathrm{sgn})$. Let $G_{\mathfrak{m}}$ and $G_{\mathfrak{m}}^{+}$be the Galois groups of $K_{\mathfrak{m}}$ and $K_{\mathfrak{m}}^{+}$over $k$, respectively. Let $J=\operatorname{Gal}\left(K_{\mathfrak{m}} / K_{\mathfrak{m}}^{+}\right) \simeq \mathbb{F}_{q}^{*}$, which is the decomposition group and inertia group of $\infty$. Let $\rho$ be a sgn-normalized Drinfeld $\mathbb{A}$-module of rank one. Then the Hilbert class field $K_{\mathfrak{e}}$ is the extension of $k$ generated by the coefficients of $\rho_{x}$ for any $x \in \mathbb{A} \backslash \mathbb{F}_{q}$. For an integral ideal $\mathfrak{m} \neq \mathfrak{e}$, let $\Lambda_{\mathfrak{m}}^{\rho}$ be the set of $\mathfrak{m}$-torsion points of $\rho$ in $\Omega$. Then the cyclotomic extension $K_{\mathfrak{m}}$ of $k$ is the extension over $K_{\mathfrak{e}}$ generated by $\Lambda_{\mathfrak{m}}^{\rho}$. Let $\xi(\mathfrak{a})$ be the $\xi$-invariant of ideal $\mathfrak{a}$, which is defined up to a constant multiplier and characterized by the condition that the $\mathbb{A}$-lattice $\xi(\mathfrak{a}) \mathfrak{a}$ corresponds to some sgn-normalized $\mathbb{A}$-module, say $\rho$. Let $e_{\mathfrak{a}}(x)$ be the Drinfeld exponential function associated to the ideal $\mathfrak{a}$. Let $\lambda_{\mathfrak{a}}=\xi(\mathfrak{a}) e_{\mathfrak{a}}(1)$. When $\mathfrak{a}$ is an integral ideal, $\lambda_{\mathfrak{a}}$ is an $\mathfrak{a}$-torsion point of $\rho$ and, in fact, it is a generator of the set $\Lambda_{\mathfrak{a}}^{\rho}$ of $\mathfrak{a}$-torsion points of $\rho$. The following are well known ([H1], [H2], or Y1. Lemma 1.2]).

Lemma 2.1. Let $\lambda$ be a generator of $\Lambda_{\mathfrak{m}}^{\rho}$ for a sgn-normalized Drinfeld $\mathbb{A}$-module $\rho$ and let $\mathfrak{p}$ be a prime ideal of $\mathbb{A}$.

(i) Assume that $\mathfrak{m}$ has at least two distinct prime divisors. Then $\lambda$ is a unit.

(ii) Assume $\mathfrak{m}=\mathfrak{p}^{n}$. Let $[\mathfrak{p}]$ be the product of the prime ideals in $\mathcal{O}_{K_{\mathrm{m}}}$ dividing $\mathfrak{p}$. Then $[\mathfrak{p}]=\lambda \mathcal{O}_{K_{\mathfrak{m}}}$.

(iii) $N_{K_{\mathfrak{p}} / K_{\mathfrak{e}}}\left(\lambda_{\mathfrak{p}}\right)=\xi(\mathbb{A}) / \xi(\mathfrak{p})$, and it generates the ideal $\mathfrak{p} \mathcal{O}_{K_{\mathfrak{e}}}$ in $\mathcal{O}_{K_{\mathfrak{e}}}$.

(iv) Let $\mathfrak{a}, \mathfrak{b}$ be fractional ideals of $\mathbb{A}$. The Galois action is

$$
(\xi(\mathbb{A}) / \xi(\mathfrak{a}))^{\tau_{\mathfrak{b}}}=\xi\left(\mathfrak{b}^{-1}\right) / \xi\left(\mathfrak{a} \mathfrak{b}^{-1}\right),
$$

where $\tau_{\mathfrak{b}}=\left(\mathfrak{b}, K_{\mathfrak{e}} / k\right)$ is the Artin symbol.

Let $F$ be a finite abelian extension of $k$ which is contained in some cyclotomic function field. Let $\mathfrak{m}$ be the conductor of $F$, i.e., $K_{\mathfrak{m}}$ is the smallest cyclotomic function field that contains $F$. If $\mathfrak{m}=\mathfrak{e}$, then $F$ is an unramified extension of $k$. For any integral ideal $\mathfrak{f}$ of $\mathbb{A}$, we let $F_{\mathfrak{f}}=F \cap K_{\mathfrak{f}}$ and $F_{\mathfrak{f}}^{+}=F \cap K_{\mathfrak{f}}^{+}$. We say that 
$F / k$ is a real extension if $F=F^{+}$. Let $G_{F}=\operatorname{Gal}(F / k)$ and $J_{F}=\operatorname{Gal}\left(F / F^{+}\right)$with $\delta_{F}=\left|J_{F}\right|$ its order. Let $\widehat{G}_{F}$ be the character group of $G_{F}$ with values in the field $\mathbb{C}$ of complex numbers. A character $\chi$ is called real if $\chi\left(J_{F}\right)=1$. Otherwise it is called non-real. We denote by $\widehat{G}_{F}^{+}$the set of all real characters of $G_{F}$ and by $\widehat{G}_{F}^{-}$ the set of all non-real characters of $G_{F}$.

Let $h(F)$ and $h\left(F^{+}\right)$be the divisor class numbers of $F$ and $F^{+}$, respectively. Let $h^{-}(F)=h(F) / h\left(F^{+}\right)$be the relative divisor class number of $F$. We have the following well-known analytic class number formulas:

$$
\begin{aligned}
h(F) & =h(k) \prod_{1 \neq \chi \in \widehat{G}_{F}} L_{k}(0, \bar{\chi}), \quad h\left(F^{+}\right)=h(k) \prod_{1 \neq \chi \in \widehat{G}_{F}^{+}} L_{k}(0, \bar{\chi}), \\
h^{-}(F) & =h(F) / h\left(F^{+}\right)=\prod_{\chi \in \widehat{G}_{F}^{-}} L_{k}(0, \chi),
\end{aligned}
$$

where $L_{k}(s, \chi)$ is the Artin $L$-function associated to the character $\chi$.

Let $\mathcal{O}_{F}$ be the integral closure of $\mathbb{A}$ in $F$ and $\mathcal{O}_{F}^{*}$ the unit group of $\mathcal{O}_{F}$. Let $h\left(\mathcal{O}_{F}\right)$ and $h\left(\mathcal{O}_{F^{+}}\right)$be the ideal class numbers of $\mathcal{O}_{F}$ and $\mathcal{O}_{F^{+}}$, respectively. Let $h^{-}\left(\mathcal{O}_{F}\right)=h\left(\mathcal{O}_{F}\right) / h\left(\mathcal{O}_{F}\right)$ be the relative ideal class number of $\mathcal{O}_{F}$. Then we have $h(F)=R(F) h\left(\mathcal{O}_{F}\right)$ and $h\left(F^{+}\right)=R\left(F^{+}\right) h\left(\mathcal{O}_{F^{+}}\right)$, where $R(F)$ and $R\left(F^{+}\right)$ are the regulators of $\mathcal{O}_{F}$ and $\mathcal{O}_{F^{+}}$, respectively. It is easy to see that $R(F)=$ $\delta_{F}^{\left[F^{+}: k\right]-1} R\left(F^{+}\right) / Q_{0}$, where $Q_{0}=\left[\mathcal{O}_{F}^{*}: \mathcal{O}_{F^{+}}^{*}\right]$. Thus we have

$$
h^{-}(F)=\delta_{F}^{\left[F^{+}: k\right]-1} h^{-}\left(\mathcal{O}_{F}\right) / Q_{0} .
$$

Lemma 2.2. $Q_{0}$ divides $\delta_{F}$.

Proof. Let $j$ be a generator of $J_{F}$. As in the proof of [Hr. Prop.1.1], $\varepsilon \mapsto \varepsilon^{1-j}=$ $\varepsilon / j(\varepsilon)$ induces an inclusion $\mathcal{O}_{F}^{*} / \mathcal{O}_{F+}^{*} \hookrightarrow \mathbb{F}_{q}^{*}$. For any $\varepsilon \in \mathcal{O}_{F}^{*}$, we have $j^{k}(\varepsilon / j(\varepsilon))=$ $\varepsilon / j(\varepsilon)$ because $\varepsilon / j(\varepsilon) \in \mathbb{F}_{q}^{*}$. Thus $\varepsilon j^{2}(\varepsilon)=j(\varepsilon)^{2}, \varepsilon^{2} j^{3}(\varepsilon)=j(\varepsilon)^{3}$ and so on. Finally we have $\varepsilon^{\delta_{F}-1} j^{\delta_{F}}(\varepsilon)=j(\varepsilon)^{\delta_{F}}$. Hence $\varepsilon^{\delta_{F}}=j\left(\varepsilon^{\delta_{F}}\right)$, i.e., $\varepsilon^{\delta_{F}} \in \mathcal{O}_{F^{+}}$. This proves the lemma.

For a subset $M$ of $G_{F}$, we set $s(M)=\sum_{\sigma \in M} \sigma$. Let $e^{+}=s\left(J_{F}\right) / \delta_{F}$ and $e^{-}=1-e^{+}$. Let $e_{\chi}=\left(1 /\left|G_{F}\right|\right) \sum_{\sigma \in G_{F}} \chi(\sigma) \sigma^{-1}$ be the idempotent associated to $\chi$. We define $\omega_{F}=\sum_{1 \neq \chi \in \widehat{G}_{F}} L_{k}(0, \bar{\chi}) e_{\chi}, \omega_{F}^{+}=e^{+} \omega_{F}$ and $\omega_{F}^{-}=e^{-} \omega_{F}$. When $F$ is the cyclotomic function field $K_{\mathfrak{f}}$, we write $\omega_{\mathfrak{f}}=\omega_{K_{\mathfrak{f}}}, \omega_{\mathfrak{f}}^{+}=\omega_{K_{\mathfrak{f}}}^{+}$and $\omega_{\mathfrak{f}}^{-}=\omega_{K_{\mathfrak{f}}}^{-}$for simplicity.

Let $l_{F}$ be the logarithm map of $F$, which is defined by

$$
l_{F}: F^{*} \rightarrow \mathbb{Q}\left[G_{F}\right], \quad x \mapsto l_{F}(x)=\sum_{\sigma \in G_{F}} v_{\infty}\left(x^{\sigma}\right) \sigma^{-1},
$$

where $v_{\infty}$ is the extension to $\Omega$ of the normalized valuation of $k_{\infty}$ at $\infty$. Let $l_{F}^{*}=\left(1-e_{1}\right) l_{F}$. When $F=K_{\mathfrak{f}}$, we write $l_{\mathfrak{f}}=l_{K_{\mathfrak{f}}}$ and $l_{\mathfrak{f}}^{*}=l_{K_{\mathfrak{f}}}^{*}$, respectively.

Suppose that $E / k$ is a finite abelian extension of $F / k$. The restriction of automorphism from $E$ to $F$ induces a ring homomorphism $\operatorname{res}_{E / F}: \mathbb{Q}\left[G_{E}\right] \rightarrow \mathbb{Q}\left[G_{F}\right]$. The corestriction map is defined as follows:

$$
\operatorname{cor}_{E / F}: \mathbb{Q}\left[G_{F}\right] \rightarrow \mathbb{Q}\left[G_{E}\right], \quad \sigma \mapsto \sum_{\tau \mapsto \sigma} \tau .
$$

Lemma 2.3. Suppose that $E / k$ is a finite abelian extension of $F / k$. Then 
(i) $l_{F}\left(N_{E / F}(x)\right)=\operatorname{res}_{E / F}\left(l_{E}(x)\right) ; l_{E}(y)=\operatorname{cor}_{E / F}\left(l_{F}(y)\right)$ for any $x \in E^{*}$ and $y \in F^{*}$;

(ii) $\operatorname{res}_{E / F}\left(\omega_{E}\right)=\omega_{F}, \operatorname{res}_{E / F}\left(\omega_{E}^{ \pm}\right)=\omega_{F}^{ \pm}$;

(iii) $\operatorname{cor}_{E / F}\left(\omega_{F}\right)=s(\operatorname{Gal}(E / F)) \omega_{E}, \operatorname{cor}_{E / F}\left(\omega_{F}^{ \pm}\right)=s(\operatorname{Gal}(E / F)) \omega_{E}^{ \pm}$.

For a character $\chi$ of $G_{F}$ and an ideal $\mathfrak{a}$ of $\mathbb{A}$, we define $\chi(\mathfrak{a}) \in \mathbb{C}$ as follows. Let $\mathfrak{f}_{\chi}$ be the conductor of $\chi$. If $\left(\mathfrak{a}, \mathfrak{f}_{\chi}\right)=\mathfrak{e}$, we let $\chi(\mathfrak{a})=\chi\left(\sigma_{\mathfrak{a}}\right)$, where $\sigma_{\mathfrak{a}}$ is the Artin $k$-automorphism of $F_{\mathfrak{f}_{\chi}}$ corresponding to $\mathfrak{a}$. If $\left(\mathfrak{a}, \mathfrak{f}_{\chi}\right) \neq \mathfrak{e}$, we put $\chi(\mathfrak{a})=0$.

For any prime ideal $\mathfrak{p}$ of $\mathbb{A}$, let $\bar{\sigma}_{\mathfrak{p}}=\mathcal{F}_{\mathfrak{p}}^{-1} s\left(T_{\mathfrak{p}}\right) /\left|T_{\mathfrak{p}}\right|$, where $T_{\mathfrak{p}}$ is the inertia group of $\mathfrak{p}$ in $G_{F}$ and $\mathcal{F}_{\mathfrak{p}}$ is a Frobenius automorphism associated to $\mathfrak{p}$ which is in the decomposition group $D_{\mathfrak{p}}$ and determined uniquely modulo $T_{\mathfrak{p}}$. Then $\chi\left(\bar{\sigma}_{\mathfrak{p}}\right)=\bar{\chi}(\mathfrak{p})$ for any $\chi \in \widehat{G}_{F}$. For any divisor $\mathfrak{f}$ of $\mathfrak{m}$, let $I_{\mathfrak{f}}=\operatorname{Gal}\left(F / F_{\mathfrak{f}}\right)$. Let $R_{F}=\mathbb{Z}\left[G_{F}\right]$. Let $V_{F}$ be the $G_{F}$-submodule of $\mathbb{Q}\left[G_{F}\right]$ generated by

$$
\alpha_{\mathfrak{f}}=s\left(I_{\mathfrak{f}}\right) \prod_{\mathfrak{p} \mid \mathfrak{f}}\left(1-\bar{\sigma}_{\mathfrak{p}}\right)
$$

with $\mathfrak{f} \mid \mathfrak{m}, \mathfrak{f} \neq \mathfrak{e}$. We also set $U_{F}=V_{F}+s\left(I_{\mathfrak{e}}\right) R_{F}$. For any $G_{F}$-module $T$, we denote by $T_{0}$ the submodule of elements of $T$ killed by $s\left(G_{F}\right)$ and for a subset $B$ of $G_{F}$, denote by $T^{B}$ the set of elements of $T$ fixed by $B$. It is easy to see that $\left(V_{F}\right)_{0}=V_{F}$ and $\left(U_{F}\right)_{0}=V_{F}+s\left(I_{\mathfrak{e}}\right)\left(R_{F}\right)_{0}$.

We conclude this section by recalling the definition of lattice index. Let $Y$ be a $\mathbb{Q}$-subspace of $\mathbb{Q}\left[G_{F}\right]$. A lattice in $Y$ is a finitely generated subgroup of $Y$ with the maximal rank. Let $L$ and $L^{\prime}$ be two lattices in $Y$. There exists a nonsingular linear transformation $A: Y \rightarrow Y$ such that $A(L)=L^{\prime}$. The lattice index is defined to be $\left(L: L^{\prime}\right)=|\operatorname{det}(A)|$. For an element $\alpha$ of $Y$, let $\left.\operatorname{ker}(\alpha)\right|_{L}$ denote the set of $x \in L$ such that $\alpha x=0$. It is known $[\mathrm{S} 2$, Lemma $1.2(\mathrm{a})]$ that

$$
\left(L: L^{\prime}\right)=\left(\left.\operatorname{ker}(\alpha)\right|_{L}:\left.\operatorname{ker}(\alpha)\right|_{L^{\prime}}\right)\left(\alpha L: \alpha L^{\prime}\right)
$$

For more properties of the lattice index, we refer to [S2 Lemma 1.1, 1.2] and Y Y3. Lemma 4.1].

\section{The Cyclotomic Units}

3.1. Cyclotomic and elliptic units. For any integral ideal $\mathfrak{f} \neq \mathfrak{e}$ of $\mathbb{A}$, we denote by $\lambda_{\mathfrak{f}}$ a primitive $\mathfrak{f}$-torsion point of a sgn-normalized Drinfeld module $\rho$ of rank one (e.g. $\left.\lambda_{\mathfrak{f}}=\xi(\mathfrak{f}) e_{\mathfrak{f}}(1)\right)$. From now on, we fix a finite abelian extension $F$ of $k$ with conductor $\mathfrak{m}$ and we let $R=R_{F}, G=G_{F}, U=U_{F}, V=V_{F}$ for simplicity. We define $\lambda_{\mathfrak{f}, F}=N_{K_{\mathfrak{f}} / F_{\mathfrak{f}}}\left(\lambda_{\mathfrak{f}}\right)$ for any ideal $\mathfrak{f} \neq \mathfrak{e}$.

Definition 3.1. Let $P_{F}$ be the $G$-submodule of $F^{*}$ generated by $\mathbb{F}_{q}^{*}$ and $\lambda_{\mathfrak{f}, F}$ with all $\mathfrak{f} \neq \mathfrak{e}$. We define $C_{F}=P_{F} \cap \mathcal{O}_{F}^{*}$, called the group of cyclotomic units of $F$.

The group of cyclotomic units $C_{F}$ has the maximal rank in $\mathcal{O}_{F}^{*}$.

Proposition 3.2. $\operatorname{rank} C_{F}=\left[F^{+}: k\right]-1$.

Proof. For any prime ideal $\mathfrak{p} \nmid \mathfrak{m}$, we have $\lambda_{\mathfrak{p}, F}=N_{K_{\mathfrak{e}} / F_{\mathfrak{e}}}(\xi(\mathbb{A}) / \xi(\mathfrak{p}))$, because $F_{\mathfrak{p}}=F_{\mathfrak{e}}$ and $N_{K_{\mathfrak{p}} / K_{\mathfrak{e}}}\left(\lambda_{\mathfrak{p}}\right)=\xi(\mathbb{A}) / \xi(\mathfrak{p})$. Now we follow the argument in [Y1, Sect. 2] to get the result.

Definition 3.3. Let $Q_{F}$ be the $G_{F_{\mathfrak{e}}}$-submodule of $F_{\mathfrak{e}}^{*}$ generated by $N_{K_{\mathfrak{e}} / F_{\mathfrak{e}}}(\xi(\mathbb{A}) /$ $\xi(\mathfrak{a}))$ with all integral ideals a. Let $E_{F}=Q_{F} \cap \mathcal{O}_{F_{\mathfrak{e}}}^{*}$, called the group of elliptic units of $F$. 
Since $N_{K_{\mathfrak{e}} / F_{\mathfrak{e}}}(\xi(\mathbb{A}) / \xi(\mathfrak{p}))=\lambda_{\mathfrak{p}, F}$ if $\mathfrak{p} \nmid \mathfrak{m}$ and $N_{K_{\mathfrak{e}} / F_{\mathfrak{e}}}(\xi(\mathbb{A}) / \xi(\mathfrak{p}))=N_{F_{\mathfrak{p}} / F_{\mathfrak{e}}}\left(\lambda_{\mathfrak{p}, F}\right)$ if $\mathfrak{p} \mid \mathfrak{m}, Q_{F}$ and $E_{F}$ are contained in $P_{F}$ and $C_{F}$, respectively.

Let $\mathbb{I}$ be the group of fractional ideals of $\mathbb{A}$ and $\mathbb{P}$ be the subgroup of principal fractional ideals of $\mathbb{A}$. Let $k_{+}$be the subgroup of $k^{*}$ consisting of positive elements.

\section{Lemma 3.4.}

(i) For any $\mathfrak{a} \in \mathbb{I}$ with $\left(\mathfrak{a}, K_{\mathfrak{e}} / k\right) \in \operatorname{Gal}\left(K_{\mathfrak{e}} / F_{\mathfrak{e}}\right)$, we have

$$
N_{K_{\mathfrak{e}} / F_{\mathfrak{e}}}(\xi(\mathbb{A}) / \xi(\mathfrak{a}))=x_{\mathfrak{a}} .
$$

Here $x_{\mathfrak{a}} \in k_{+}$denotes the unique element such that $\mathfrak{a}^{\left[K_{\mathfrak{e}}: F_{\mathfrak{e}}\right]}=x_{\mathfrak{a}} \mathbb{A}$.

(ii) $Q_{F} \cap k=\left\{x_{\mathfrak{a}} \in k_{+}: \mathfrak{a} \in \mathbb{I}\right.$ with $\left.\left(\mathfrak{a}, K_{\mathfrak{e}} / k\right) \in \operatorname{Gal}\left(K_{\mathfrak{e}} / F_{\mathfrak{e}}\right)\right\}$.

Proof. (i) Let $Q$ be the $G_{\mathfrak{e}}$-submodule of $K_{\mathfrak{e}}^{*}$ generated by $\xi(\mathbb{A}) / \xi(\mathfrak{a})$ for all $\mathfrak{a} \in \mathbb{I}$. Let $\mathcal{N}=\operatorname{Gal}\left(K_{\mathfrak{e}} / F_{\mathfrak{e}}\right)$ and let $Q_{\mathcal{N}}$ be the $G_{\mathfrak{e}}$-submodule of $Q$ generated by $\xi(\mathbb{A}) / \xi(\mathfrak{a})$ with all $\left(\mathfrak{a}, K_{\mathfrak{e}} / k\right) \in \mathcal{N}$. Then we have the following exact sequence ([H1, Sect. 4]):

$$
1 \longrightarrow Q_{\mathcal{N}} / k_{+} \longrightarrow Q / k_{+} \stackrel{N_{K_{\mathfrak{e}} / F_{\mathfrak{e}}}}{\longrightarrow} Q \cap F_{\mathfrak{e}} / k_{+} \longrightarrow 1 .
$$

For any $\mathfrak{a} \in \mathbb{I}$ with $\left(\mathfrak{a}, K_{\mathfrak{e}} / k\right) \in \mathcal{N}$, we have (by Lemma 2.1 (iv)),

$$
\begin{aligned}
N_{K_{\mathfrak{e}} / F_{\mathfrak{e}}}(\xi(\mathbb{A}) / \xi(\mathfrak{a} \mathfrak{b})) & =N_{K_{\mathfrak{e}} / F_{\mathfrak{e}}}(\xi(\mathbb{A}) / \xi(\mathfrak{a})) N_{K_{\mathfrak{e}} / F_{\mathfrak{e}}}(\xi(\mathbb{A}) / \xi(\mathfrak{b})), \\
N_{K_{\mathfrak{e}} / F_{\mathfrak{e}}}\left((\xi(\mathbb{A}) / \xi(\mathfrak{a}))^{\sigma}\right) & =N_{K_{\mathfrak{e}} / F_{\mathfrak{e}}}(\xi(\mathbb{A}) / \xi(\mathfrak{a})),
\end{aligned}
$$

for any $\mathfrak{b} \in \mathbb{I}$ and $\sigma \in G_{\mathfrak{e}}$. Let $x=N_{K_{\mathfrak{e}} / F_{\mathfrak{e}}}(\xi(\mathbb{A}) / \xi(\mathfrak{a}))$ with $\left(\mathfrak{a}, K_{\mathfrak{e}} / k\right) \in \mathcal{N}$. Let $n_{F}=\left[K_{\mathfrak{e}}: F_{\mathfrak{e}}\right]$. Since $\mathfrak{a}^{n_{F}}=x_{\mathfrak{a}} \mathbb{A}$, we have $x_{\mathfrak{a}}=\xi(\mathbb{A}) / \xi\left(\mathfrak{a}^{n_{F}}\right)$. Thus we have (by $(3.2)$ )

$$
x_{\mathfrak{a}}^{n_{F}}=N_{K_{\mathfrak{e}} / F_{\mathfrak{e}}}\left(\xi(\mathbb{A}) / \xi\left(\mathfrak{a}^{n_{F}}\right)\right)=\left(N_{K_{\mathfrak{e}} / F_{\mathfrak{e}}}(\xi(\mathbb{A}) / \xi(\mathfrak{a}))\right)^{n_{F}}=x^{n_{F}} .
$$

But $x$ and $x_{\mathfrak{a}}$ are positive; so we have $x=x_{\mathfrak{a}}$.

(ii) We claim that $x \in k \cap Q_{F}$ if and only if $x=N_{K_{\mathfrak{e}} / F_{\mathfrak{e}}}(\xi(\mathbb{A}) / \xi(\mathfrak{a}))$ for some $\mathfrak{a} \in \mathbb{I}$ with $\left(\mathfrak{a}, K_{\mathfrak{e}} / k\right) \in \mathcal{N}$. For $x \in k \cap Q_{F}, x=N_{K_{\mathfrak{e}} / F_{\mathfrak{e}}}(y)$ for some $y \in Q$. By (3.1), we can write $y=y_{1} y_{2}$ where $y_{1}=\prod_{i}\left(\xi(\mathbb{A}) / \xi\left(\mathfrak{a}_{i}\right)\right)^{a_{i}} \in Q_{\mathcal{N}}$ with $\left(\mathfrak{a}_{i}, K_{\mathfrak{e}} / k\right) \in \mathcal{N}$ and $y_{2} \in k_{+}$. Then, by (3.2) and (3.3), we have $x=N_{K_{\mathfrak{e}} / F_{\mathfrak{e}}}(\xi(\mathbb{A}) / \xi(\mathfrak{a}))$ where $\mathfrak{a}=\prod_{i} \mathfrak{a}_{i}^{\operatorname{deg} a_{i}} y_{2} \mathbb{A}$ and $\left(\mathfrak{a}, K_{\mathfrak{e}} / k\right) \in \mathcal{N}$. The converse follows directly from (3.3).

Lemma 3.5. $\left(Q_{F} / E_{F}\right) /\left(Q_{F} \cap k\right) \simeq G_{F_{\mathrm{e}}}$.

Proof. Let $Q$ be the $G_{\mathfrak{e}}$-submodule of $K_{\mathfrak{e}}^{*}$ defined in the proof of Lemma 3.4 and $E=Q \cap \mathcal{O}_{K_{\mathrm{e}}}^{*}$. Let $\phi: \mathbb{I} \rightarrow Q / E$ be the homomorphism defined by $\phi(\mathfrak{a})=$ $\prod_{\mathfrak{p}}(\xi(\mathbb{A}) / \xi(\mathfrak{p}))^{\operatorname{ord}_{\mathfrak{p}} \mathfrak{a}} \bmod E$. It is easy to show that $\phi$ is an isomorphism and $\phi^{-1}(Q \cap k)=\mathbb{P}$. Here we view $Q \cap k$ as a subgroup of $Q / E$. Let $\psi: \mathbb{I} \rightarrow G_{\mathfrak{e}}$

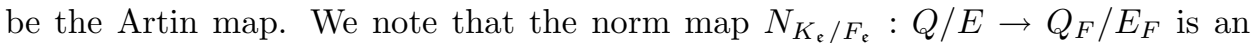
isomorphism. Let $\alpha=\psi \circ \phi^{-1}$ and $\alpha_{F}=\operatorname{res}_{K_{\mathrm{e}} / F_{\mathrm{e}}} \circ \alpha \circ N_{K_{\mathrm{e}} / F_{\mathrm{e}}}^{-1}$. We also view $Q_{F} \cap k$ as a subgroup of $Q_{F} / E_{F}$. We want to show that $Q_{F} \cap k=\operatorname{ker} \alpha_{F}$. Let $x \in Q_{F} \cap k$. Then, by Lemma 3.4 (ii), we have $x=N_{K_{\mathrm{e}} / F_{\mathrm{e}}}(\xi(\mathbb{A}) / \xi(\mathfrak{a}))$ with $\left(\mathfrak{a}, K_{\mathfrak{e}} / k\right) \in \operatorname{Gal}\left(K_{\mathfrak{e}} / F_{\mathfrak{e}}\right)$. Thus $\alpha_{F}(x)=\operatorname{res}_{K_{\mathfrak{e}} / F_{\mathfrak{e}}}\left(\left(\mathfrak{a}, K_{\mathfrak{e}} / k\right)\right)$, which is trivial in $G_{F_{\mathfrak{e}}}$. Conversely if $\xi(\mathbb{A}) / \xi(\mathfrak{a}) \in N_{K_{\mathfrak{e}} / F_{\mathfrak{e}}}^{-1}\left(\operatorname{ker}\left(\alpha_{F}\right)\right)$, then $\alpha(\xi(\mathbb{A}) / \xi(\mathfrak{a}))=\left(\mathfrak{a}, K_{\mathfrak{e}} / k\right) \in$ $\operatorname{Gal}\left(K_{\mathfrak{e}} / F_{\mathfrak{e}}\right)$, and so $N_{K_{\mathfrak{e}} / F_{\mathfrak{e}}}(\xi(\mathbb{A}) / \xi(\mathfrak{a}))=x_{\mathfrak{a}} \in Q_{F} \cap k$ (by Lemma 3.4 (i), (ii)). 
Therefore we have $\operatorname{ker}\left(\alpha_{F}\right)=Q_{F} \cap k$ and the following commutative diagram:

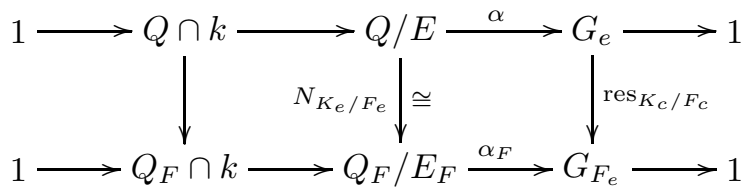

with exact rows. Thus we get the result.

3.2. Calculation of $\left[\mathcal{O}_{F}^{*}: C_{F}\right]$. Let $\mathfrak{m}=\mathfrak{p}_{1}^{e_{1}} \mathfrak{p}_{2}^{e_{2}} \cdots \mathfrak{p}_{s}^{e_{s}}$ be the conductor of $F$. Let $\omega_{F}^{*}=(q-1) \omega_{F}^{+}$and $\omega_{f}^{*}=(q-1) \omega_{f}^{+}$. It is known [Y1, Prop. 4.1] that $l_{f}^{*}\left(\lambda_{\mathfrak{f}}\right)=$ $\omega_{\mathfrak{f}}^{*} \prod_{\mathfrak{p} \mid \mathfrak{f}}\left(1-\bar{\sigma}_{\mathfrak{p}}\right) \in \mathbb{Q}\left[\operatorname{Gal}\left(K_{\mathfrak{f}} / k\right)\right]$. This fact and $\operatorname{cor}_{F / F_{\mathfrak{f}}} \operatorname{res}_{K_{\mathfrak{f}} / F_{\mathfrak{f}}}\left(\omega_{\mathfrak{f}}^{*}\right)=s\left(I_{\mathfrak{o}}\right) \omega_{F}^{*}$ imply

$$
l_{F}^{*}\left(\lambda_{\mathfrak{f}, F}\right)=\omega_{F}^{*} s\left(I_{\mathfrak{d}}\right) \prod_{\substack{\mathfrak{p} \mid \mathfrak{f} \\ \mathfrak{p} \nmid \mathfrak{m}}}\left(1-\mathcal{F}_{\mathfrak{p}}^{-1}\right) \prod_{\mathfrak{p} \mid \mathfrak{d}}\left(1-\bar{\sigma}_{\mathfrak{p}}\right)
$$

where $\mathfrak{d}=(\mathfrak{f}, \mathfrak{m})$. Thus we have

Proposition 3.6. $l_{F}^{*}\left(P_{F}\right)=\omega_{F}^{*} U_{0}$.

Let $V_{1}$ be the $G$-submodule of $V$ consisting of all the sum $\sum_{\mathfrak{e} \neq \mathfrak{f} \mid \mathfrak{m}} a_{\mathfrak{f}} \alpha_{\mathfrak{f}}$, where the coefficients $a_{\mathfrak{f}} \in R$ with $\sum_{\mathfrak{n} \mid \mathfrak{p}_{i}^{e_{i}}}\left[F_{\mathfrak{p}_{i} e_{i}}: F_{\mathfrak{n}}\right] a_{\mathfrak{n}} \in R_{0}$ for all $i \in\{1,2, \ldots, s\}$.

Proposition 3.7. $l_{F}\left(C_{F}\right)=\omega_{F}^{*}\left(V_{1}+s\left(I_{\mathfrak{e}}\right) R_{0}^{2}\right)$.

Proof. For any $x \in P_{F}$, we can write it in the form

$$
x=c \prod_{\mathfrak{f}} \lambda_{\mathfrak{f}, F}^{a_{\mathfrak{f}}} \prod_{\mathfrak{p} \nmid \mathfrak{m}} \lambda_{\mathfrak{p}, F}^{a_{\mathfrak{p}}} \prod_{i=1}^{s} \prod_{\mathfrak{n} \mid \mathfrak{p}_{i}^{e_{i}}} \lambda_{\mathfrak{n}, F}^{a_{\mathfrak{n}}},
$$

where $\mathfrak{f}$ runs through non-prime-power ideals and $c \in \mathbb{F}_{q}^{*}, a_{\mathfrak{f}}, a_{\mathfrak{p}}, a_{\mathfrak{n}} \in R$. Then $x \in C_{F}$ if and only if $a_{\mathfrak{p}} \in R_{0}$ with all primes $\mathfrak{p} \nmid \mathfrak{m}$ and $\sum_{\mathfrak{n} \mid \mathfrak{p}_{i}^{e_{i}}}\left[F_{\mathfrak{p}_{i}}^{e_{i}}: F_{\mathfrak{n}}\right] a_{\mathfrak{n}} \in R_{0}$ for $i=1,2, \ldots, s$. Note that $l_{F}^{*}\left(\lambda_{\mathfrak{p}, F}\right)=\omega_{F}^{*} s\left(I_{\mathfrak{e}}\right)\left(1-\mathcal{F}_{\mathfrak{p}}^{-1}\right) \in \omega_{F}^{*} s\left(I_{\mathfrak{e}}\right) R_{0}$ with all primes $\mathfrak{p} \nmid \mathfrak{m}$. Thus $l_{F}^{*}\left(\lambda_{\mathfrak{p}, F}^{a_{\mathfrak{p}}}\right) \in \omega_{F}^{*} s\left(I_{\mathfrak{e}}\right) R_{0}^{2}$. For a non-prime-power ideal $\mathfrak{f}$, we have

$$
l_{F}^{*}\left(\lambda_{\mathfrak{f}, F}\right)=\omega_{F}^{*} s\left(I_{\mathfrak{d}}\right) \prod_{\substack{\mathfrak{p} \mid \mathfrak{f} \\ \mathfrak{p} \nmid \mathfrak{m}}}\left(1-\mathcal{F}_{\mathfrak{p}}^{-1}\right) \prod_{\mathfrak{p} \mid \mathfrak{d}}\left(1-\bar{\sigma}_{\mathfrak{p}}\right),
$$

where $\mathfrak{d}=(\mathfrak{f}, \mathfrak{m})$. If $\mathfrak{d}=\mathfrak{e}, l_{F}^{*}\left(\lambda_{\mathfrak{f}, F}\right) \in \omega_{F}^{*} s\left(I_{\mathfrak{e}}\right) R_{0}^{2}$. If $\mathfrak{d} \neq \mathfrak{e}$ is divisible by only one prime (say, $\mathfrak{d}=\mathfrak{p}_{i}^{z_{i}}$ with $z_{i} \leq e_{i}$ ), then $l_{F}^{*}\left(\lambda_{\mathfrak{f}, F}\right) \in \omega_{F}^{*} \alpha_{\mathfrak{p}_{i} z_{i}} R_{0} \subset \omega_{F}^{*} V_{1}$. For composite $\mathfrak{d}$, there is no required condition. Thus we get the result.

Proposition 3.8. Let $F / k$ be a finite abelian extension with conductor $\mathfrak{m}=$ $\mathfrak{p}_{1}^{e_{1}} \mathfrak{p}_{2}^{e_{2}} \cdots \mathfrak{p}_{s}^{e_{s}}$. Then we have

$$
\left[P_{F}^{q-1} \cap k: Q_{F}^{q-1} \cap k\right]\left[l_{F}^{*}\left(P_{F}\right): l_{F}\left(C_{F}\right)\right]=\left[F_{\mathfrak{e}}: k\right] \prod_{i=1}^{s}\left[F_{\mathfrak{p}_{i}^{e_{i}}}: F_{\mathfrak{e}}\right] .
$$

Moreover, $\left[P_{F}^{q-1} \cap k: Q_{F}^{q-1} \cap k\right]=1$ if $F$ satisfies one of the following four conditions:

(i) $F$ is a real extension of $k$ that contains the Hilbert class field $K_{\mathfrak{e}}$;

(ii) $\operatorname{Gal}\left(F / F_{\mathfrak{e}}\right)$ is cyclic;

(iii) $\operatorname{Gal}\left(F / F_{\mathfrak{e}}\right)$ is the direct product of the inertia groups $T_{\mathfrak{p}_{i}}$; 
(iv) $s \leq 2$.

Proof. The main idea of the proof of (3.5) is due to Linsheng Yin ([Y1, Prop. 5.1]). From the exact sequence $0 \rightarrow \mathbb{F}_{q}^{*} \rightarrow \operatorname{ker}\left(l_{F}^{*}\right) \cap P_{F} \rightarrow P_{F} / C_{F} \rightarrow l_{F}^{*}\left(P_{F}\right) / l_{F}\left(C_{F}\right) \rightarrow 0$, we have

$$
\left[l_{F}^{*}\left(P_{F}\right): l_{F}\left(C_{F}\right)\right]=\left[P_{F} / C_{F}: \operatorname{ker}\left(l_{F}^{*}\right) \cap P_{F} / \mathbb{F}_{q}^{*}\right] .
$$

For each prime ideal $\mathfrak{p}$ of $\mathbb{A}$, choose a prime ideal $\wp$ of $\mathcal{O}_{F_{\mathrm{e}}}$ and a prime ideal $\Re$ of $\mathcal{O}_{F}$ lying over $\mathfrak{p}$. Define $v_{F}: P_{F} \rightarrow \bigoplus_{\Re} \mathbb{Z}$ by $v_{F}(x)=\left(v_{\Re}(x)\right)_{\Re}$ and $v_{F_{\mathfrak{e}}}: Q_{F} \rightarrow \bigoplus_{\wp} \mathbb{Z}$ by $v_{F_{\mathrm{e}}}(y)=\left(v_{\wp}(y)\right)_{\wp}$. Then we have the exact sequences

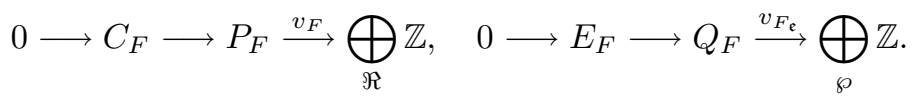

It is easy to check that $\operatorname{Im} v_{F}=\bigoplus_{\Re}\left[K_{\mathfrak{e}}: F_{\mathfrak{e}}\right] \mathbb{Z}$ and $\operatorname{Im} v_{F_{\mathfrak{e}}}=\bigoplus_{\wp}\left[K_{\mathfrak{e}}: F_{\mathfrak{e}}\right] \mathbb{Z}$. Define $e_{F / F_{\mathrm{e}}}: \operatorname{Im} v_{F_{\mathrm{e}}} \rightarrow \operatorname{Im} v_{F}$ by multiplying the $\wp$-th component by the ramification index $e_{\Re / \wp}$ for all $\wp$. Then we have the following commutative diagram:

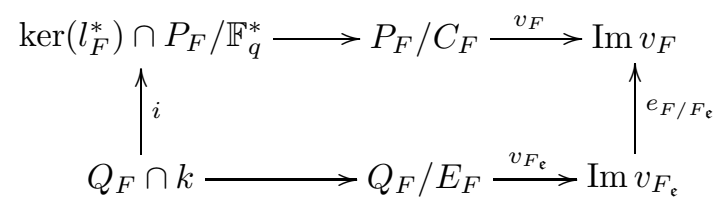

where $i$ is induced by the inclusion. Since $e_{\Re_{i} / \wp_{i}}=\left[F_{\mathfrak{p}_{i}}: F_{\mathfrak{e}}\right]$ for $i=1,2, \ldots, s$ and $e_{\Re / \wp}=1$ for all $\mathfrak{p} \nmid \mathfrak{m}$, we have $\left[\operatorname{Im} v_{F}: e_{F / F_{\mathfrak{e}}}\left(\operatorname{Im} v_{F_{\mathfrak{e}}}\right)\right]=\prod_{i=1}^{s}\left[F_{\mathfrak{p}_{i} e_{i}}: F_{\mathfrak{e}}\right]$. By (3.6), Lemma 3.5 and the above commutative diagram, we have

$$
\left[\operatorname{ker}\left(l_{F}^{*}\right) \cap P_{F} / \mathbb{F}_{q}^{*}: Q_{F} \cap k\right]\left[l_{F}^{*}\left(P_{F}\right): l_{F}\left(C_{F}\right)\right]=\left[F_{\mathfrak{e}}: k\right] \prod_{i}\left[F_{\mathfrak{p}_{i} e_{i}}: F_{\mathfrak{e}}\right] .
$$

We note that any element of $P_{F}^{q-1}$ is positive. As in the proof of [Y1, Lemma 4.5], we have $\operatorname{ker}\left(l_{F}^{*}\right) \cap P_{F}^{q-1}=P_{F}^{q-1} \cap k$. Then the surjective homomorphism $\operatorname{ker}\left(l_{F}^{*}\right) \cap P_{F} \rightarrow$ $P_{F}^{q-1} \cap k$ defined by $x \mapsto x^{q-1}$ induces an isomorphism $\left(\operatorname{ker}\left(l_{F}^{*}\right) \cap P_{F} / \mathbb{F}_{q}^{*}\right) / Q_{F} \cap k \simeq$ $P_{F}^{q-1} \cap k / Q_{F}^{q-1} \cap k$. Thus we have $\left[\operatorname{ker}\left(l_{F}^{*}\right) \cap P_{F} / \mathbb{F}_{q}^{*}: Q_{F} \cap k\right]=\left[P_{F}^{q-1} \cap k: Q_{F}^{q-1} \cap k\right]$.

If $F$ is a real extension of $k$ that contains the Hilbert class field $K_{\mathfrak{e}}$, then $Q_{F}$ is just $Q$ and so $Q_{F} \cap k=k_{+}$([H1, Cor. 2.5]). Since $F / k$ is a real extension, any element of $P_{F}$ is positive and so $\operatorname{ker}\left(l_{F}^{*}\right) \cap P_{F}=k^{*}$ as in the proof of [Y1, Lemma 4.5]. Thus we have $\left[\operatorname{ker}\left(l_{F}^{*}\right) \cap P_{F} / \mathbb{F}_{q}^{*}: Q_{F} \cap k\right]=1$.

Now assume that $F$ satisfies one of conditions (ii), (iii) or (iv) in the proposition. We calculate the index $\left[l_{F}^{*}\left(P_{F}\right): l_{F}\left(C_{F}\right)\right]$ in a different way (which is due to Hassan Oukhaba). By Propositions 3.6 and 3.7 we have

$$
\left[l_{F}^{*}\left(P_{F}\right): l_{F}\left(C_{F}\right)\right]=\left[\omega_{F}^{*} U_{0}: \omega_{F}^{*}\left(V_{1}+s\left(I_{\mathfrak{e}}\right) R_{0}^{2}\right)\right]=\left[U_{0}: V_{1}+s\left(I_{\mathfrak{e}}\right) R_{0}^{2}\right] .
$$

The last equality follows from the fact that $\omega_{F}^{*}$ is an automorphism of $e^{+} \mathbb{C}[G]_{0}$. We define a mapping $\Theta: U_{0} \rightarrow \prod_{i=1}^{s} \mathbb{Z} /\left[F_{\mathfrak{p}_{i} e_{i}}: F_{\mathfrak{e}}\right] \mathbb{Z}$ by

$$
\Theta\left(\sum_{\mathfrak{e} \neq \mathfrak{n} \mid \mathfrak{m}} a_{\mathfrak{n}} \alpha_{\mathfrak{n}}+a_{\mathfrak{e}} s\left(I_{\mathfrak{e}}\right)\right)=\left(\sum_{\mathfrak{n} \mid \mathfrak{p}_{i}^{e_{i}}}\left[F_{\mathfrak{p}_{i}^{e_{i}}}: F_{\mathfrak{n}}\right] \operatorname{deg}\left(a_{\mathfrak{n}}\right) \quad \bmod \left[F_{\mathfrak{p}_{i}^{e_{i}}}: F_{\mathfrak{e}}\right]\right)_{i} .
$$


In Corollary $3.12(\mathrm{i})$, it is shown that $\Theta$ is a well-defined surjective homomorphism with $\operatorname{ker}(\Theta)=V_{1}+s\left(I_{\mathfrak{e}}\right) R_{0}$. Thus we have

$$
\left[U_{0}: V_{1}+s\left(I_{\mathfrak{e}}\right) R_{0}\right]=\prod_{i=1}^{s}\left[F_{\mathfrak{p}_{i} e_{i}}: F_{\mathfrak{e}}\right] .
$$

Let $I_{(1)}$ be the augmentation ideal of $\mathbb{Z}\left[G_{F_{\mathrm{e}}}\right]$. We define a mapping $\mathcal{G}: V_{1}+$ $s\left(I_{\mathfrak{e}}\right) R_{0} \rightarrow I_{(1)}$ by $\mathcal{G}\left(\sum_{\mathfrak{e} \neq \mathfrak{n} \mid \mathfrak{m}} a_{\mathfrak{n}} \alpha_{\mathfrak{n}}+a_{\mathfrak{e}} s\left(I_{\mathfrak{e}}\right)\right)=\operatorname{res}_{F / F_{\mathfrak{e}}}\left(a_{\mathfrak{e}}\right)$, which is a well-defined surjective homomorphism and $\mathcal{G}^{-1}\left(I_{(1)}^{2}\right)=V_{1}+s\left(I_{\mathfrak{e}}\right) R_{0}^{2}$ (Cor. 3.12 (ii)). Thus we have

$$
\left[V_{1}+s\left(I_{\mathfrak{e}}\right) R_{0}: V_{1}+s\left(I_{\mathfrak{e}}\right) R_{0}^{2}\right]=\left[I_{(1)}: I_{(1)}^{2}\right]=\left|G_{F_{\mathfrak{e}}}\right| .
$$

By combining (3.7), (3.8) and (3.9), we have $\left[l_{F}^{*}\left(P_{F}\right): l_{F}\left(C_{F}\right)\right]=\left[F_{\mathfrak{e}}: k\right] \prod_{i}\left[F_{\mathfrak{p}_{i}}{ }_{i}:\right.$ $\left.F_{\mathfrak{e}}\right]$ and so $\left[P_{F}^{q-1} \cap k: Q_{F}^{q-1} \cap k\right]=1$.

We calculate the index $\left[\mathcal{O}_{F}^{*}: C_{F}\right]$. Note that $\operatorname{ker}\left(l_{F}\right) \cap \mathcal{O}_{F}^{*}=\mathbb{F}_{q}^{*}=\operatorname{ker}\left(l_{F}\right) \cap C_{F}^{*}$. Write the index $\left[\mathcal{O}_{F}^{*}: C_{F}\right]$ as follows:

$\left[\mathcal{O}_{F}^{*}: C_{F}\right]=\left[l_{F}\left(\mathcal{O}_{F}^{*}\right): l_{F}\left(C_{F}\right)\right]=\left(l_{F}\left(\mathcal{O}_{F}^{*}\right): e^{+} R_{0}\right)\left(e^{+} R_{0}: e^{+} U_{0}\right)\left(e^{+} U_{0}: l_{F}^{*}\left(P_{F}\right)\right)$

$$
\times\left(l_{F}^{*}\left(P_{F}\right): l_{F}\left(C_{F}\right)\right) .
$$

We compute the indices respectively.

Since $\left(e^{+} R_{0}: l_{F}\left(\mathcal{O}_{F}^{*}\right)\right)=R(F)$ and $R(F)=\delta_{F}^{\left[F^{+}: k\right]-1} R\left(F^{+}\right) / Q_{0}$, we have

$$
\left(l_{F}\left(\mathcal{O}_{F}^{*}\right): e^{+} R_{0}\right)=\delta_{F}^{1-\left[F^{+}: k\right]} Q_{0} / R\left(F^{+}\right) .
$$

Since $l_{F}^{*}\left(P_{F}\right)=\omega_{F}^{*} U_{0}=\omega_{F}^{*} e^{+} U_{0}$ and $h\left(F^{+}\right)=R\left(F^{+}\right) h\left(\mathcal{O}_{F^{+}}\right)$, we have

$$
\begin{aligned}
& \left(e^{+} U_{0}: l_{F}^{*}\left(P_{F}\right)\right)=\operatorname{det} \omega_{F}^{*}=\prod_{1 \neq \chi \in \widehat{G}_{F}^{+}}(q-1) L_{k}(0, \bar{\chi}) \\
& =(q-1)^{\left[F^{+}: k\right]-1} R\left(F^{+}\right) h\left(\mathcal{O}_{F^{+}}\right) / h(k) .
\end{aligned}
$$

Since $\left(e^{+} R: e^{+} U\right)=\left(s(G) e^{+} R: s(G) e^{+} U\right)\left(e^{+} R_{0}: e^{+} U_{0}\right), s(G) e^{+} R=\mathbb{Z} s(G)$ and $s(G) e^{+} U=\left|I_{\mathfrak{e}}\right| \mathbb{Z} s(G)$, we have

$$
\left(e^{+} R_{0}: e^{+} U_{0}\right)=\left(e^{+} R: e^{+} U\right) /\left[F: F_{\mathbf{e}}\right] .
$$

By substituting (3.11), (3.12) and (3.13) into (3.10) and by Proposition 3.8 we have the following theorem.

Theorem 3.9. Let $F / k$ be a finite abelian extension with conductor $\mathfrak{m}=\mathfrak{p}_{1}^{e_{1}} \cdots \mathfrak{p}_{s}^{e_{s}}$. Let us put $d(F)=\left[P_{F}^{q-1} \cap k: Q_{F}^{q-1} \cap k\right]$. Then we have

$$
\left[\mathcal{O}_{F}^{*}: C_{F}\right]=\left((q-1) / \delta_{F}\right)^{\left[F^{+}: k\right]-1} Q_{0} h\left(\mathcal{O}_{F^{+}}\right) \frac{\prod_{i=1}^{s}\left[F_{\mathfrak{p}_{i} e_{i}}: F_{\mathfrak{e}}\right]}{\left[F: F_{\mathfrak{e}}\right]\left[K_{\mathfrak{e}}: F_{\mathfrak{e}}\right]} \frac{\left(e^{+} R: e^{+} U\right)}{d(F)} .
$$

Remark 3.10. In [Hr. Thm. 3.9], Harrop has calculated the same index in the rational function field case. But his formula is not consistent with ours. This inconsistency is mainly due to his incorrect equality $\left[T_{1}^{G}: l\left(\mathbb{F}_{q}(T)^{*}\right)\right]=\left|\left(D / \mathbb{F}_{q}(T)^{*}\right)_{q-1}\right|$ in [Hr, Lemma 3.4] because ker $l$ is not contained in $\mathbb{F}_{q}(T)$. The equation in [Hr. Prop. 3.6] should be replaced by $\left[\left(1-e_{1}\right) T_{1}: l(C)\right]=\left|\left(D / \mathbb{F}_{q}(T)^{*}\right)_{q-1}\right|^{-1} \prod_{i=1}^{g}\left[k_{Q_{i}^{e_{i}}}\right.$ : $\left.\mathbb{F}_{q}(T)\right]$. In [Hr, Prop. 3.7], $\omega^{\prime}$ should be replaced by $\sum_{\chi \neq \chi_{0}} \varphi_{F_{\chi}}(\bar{\chi}) e_{\chi} /|J \cap I|$, and so the equation in $[\mathrm{Hr}$, page 420 , line 21$]$ should be replaced by $\left(e^{+} U_{0}:\left(1-e_{1}\right) T_{1}\right)=$ 
$(q-1)^{2 r}|J \cap I|^{-r} h\left(k^{+}\right)$. Finally, one should replace $|J \cap I|^{2 r}$ by $|J \cap I|^{r}$ in $[\mathrm{Hr}$. Thm. 3.9].

3.3. Generators and relations for $U$. In this subsection we show that $\Theta$ and $\mathcal{G}$ defined in the proof of Proposition 3.8 are well defined and have the desired properties. The main ideas of this subsection are due to Hassan Oukhaba and Jean-Robert Belliard [B, BO, O]. Recall that $\mathfrak{m}=\mathfrak{p}_{1}^{e_{1}} \cdots \mathfrak{p}_{s}^{e_{s}}$ is the conductor of $F$. Let $\Omega=\left\{\left(t_{1}, \ldots, t_{s}\right): t_{i}=0\right.$ or 1 for all $\left.i=1, \ldots, s\right\}$. For $t=\left(t_{1}, \ldots, t_{s}\right) \in \Omega$, let $\operatorname{supp}(t)$ be the set of $i \in\{1, \ldots, s\}$ such that $t_{i}=1$. In particular, we denote by $\langle i\rangle$ the unique element $t$ of $\Omega$ such that $\operatorname{supp}(t)=\{i\}$. Let $\mathbb{G}$ be the free abelian group on the disjoint union $\coprod_{t \in \Omega} \operatorname{Gal}\left(F_{\mathfrak{n}_{t}} / k\right)$, where $\mathfrak{n}_{t}=\mathfrak{p}_{1}^{t_{1} e_{1}} \cdots \mathfrak{p}_{s}^{t_{s}} e_{s}$ for $t=\left(t_{1}, \ldots, t_{s}\right) \in \Omega$. Put $\alpha_{\mathfrak{e}}=s\left(I_{\mathfrak{e}}\right)$. Since $\left\{\alpha_{\mathfrak{n}_{t}}\right\}_{t \in \Omega}$ generates all $\alpha_{\mathfrak{n}}$ with $\mathfrak{n} \mid \mathfrak{m}$ over $R$, we obtain a surjective homomorphism $\mathcal{F}: \mathbb{G} \rightarrow U$ by mapping $\sigma \in \operatorname{Gal}\left(F_{\mathfrak{n}_{t}} / k\right)$ to $\tilde{\sigma} \alpha_{\mathfrak{n}_{t}}$, where $\tilde{\sigma}$ is any extension of $\sigma$ to $G$.

Suppose we have $t \in \Omega$ and $i \in \operatorname{supp}(t)$. Let $\sigma \in \operatorname{Gal}\left(F_{\mathfrak{n}_{t}(i)} / k\right)$ where $\mathfrak{n}_{t}(i)=$ $\mathfrak{n}_{t} \mathfrak{p}_{i}^{-e_{i}}$. Let $\tilde{\sigma} \in \operatorname{Gal}\left(F_{\mathfrak{n}_{t}} / k\right)$ be an extension of $\sigma$ to $F_{\mathfrak{n}_{t}}$. Then, as one may check easily, the formal sums

$$
S(t, i, \sigma)=s\left(\operatorname{Gal}\left(F_{\mathfrak{n}_{t}} / F_{\mathfrak{n}_{t}(i)}\right)\right) \tilde{\sigma}-\left(1-\left(\mathfrak{p}_{i}, F_{\mathfrak{n}_{t}(i)} / k\right)^{-1}\right) \sigma
$$

are all contained in ker $\mathcal{F}$.

Since the proof of the following theorem is almost the same as the ones in $\mathrm{BO}$. Prop. 3.6] and [B, Prop. 4.1, 4.6], we leave it to the readers.

Theorem 3.11. Suppose that $F / k$ satisfies one of the following conditions:

(i) $\operatorname{Gal}\left(F / F_{\mathfrak{e}}\right)$ is cyclic;

(ii) $\operatorname{Gal}\left(F / F_{\mathfrak{e}}\right)$ is the direct product of the inertia groups $T_{\mathfrak{p}_{i}}$;

(iii) $s \leq 2$.

Then ker $\mathcal{F}$ is generated by $S(t, i, \sigma)$ with $t \in \Omega, i \in \operatorname{supp}(t), \sigma \in \operatorname{Gal}\left(F_{\mathfrak{n}_{t}(i)} / k\right)$.

Corollary 3.12. Suppose that $F / k$ satisfies one of the conditions in Theorem 3.11 .

(i) Let $\Theta: U_{0} \rightarrow \prod_{i=1}^{s} \mathbb{Z} /\left[F_{\mathfrak{p}_{i}}: F_{\mathfrak{e}}\right] \mathbb{Z}$ be defined by

$$
\Theta\left(\sum_{\mathfrak{e} \neq \mathfrak{n} \mid \mathfrak{m}} a_{\mathfrak{n}} \alpha_{\mathfrak{n}}+a_{\mathfrak{e}} s\left(I_{\mathfrak{e}}\right)\right)=\left(\sum_{\mathfrak{n} \mid \mathfrak{p}_{i}^{e_{i}}}\left[F_{\mathfrak{p}_{i}^{e_{i}}}: F_{\mathfrak{n}}\right] \operatorname{deg} a_{\mathfrak{n}} \bmod \left[F_{\mathfrak{p}_{i}^{e_{i}}}: F_{\mathfrak{e}}\right]\right)_{i} .
$$

Then it is a well-defined surjective homomorphism with $\operatorname{ker} \Theta=V_{1}+$ $s\left(I_{\mathfrak{e}}\right) R_{0}$.

(ii) Let $I_{(1)}$ be the augmentation ideal of $\mathbb{Z}\left[G_{F_{\mathfrak{e}}}\right]$. Let $\mathcal{G}: V_{1}+s\left(I_{\mathfrak{e}}\right) R_{0} \rightarrow I_{(1)}$ be defined by $\mathcal{G}\left(\sum_{\mathfrak{e} \neq \mathfrak{n} \mid \mathfrak{m}} a_{\mathfrak{n}} \alpha_{\mathfrak{n}}+a_{\mathfrak{e}} s\left(I_{\mathfrak{e}}\right)\right)=\operatorname{res}_{F / F_{\mathfrak{e}}}\left(a_{\mathfrak{e}}\right)$. Then it is a welldefined surjective homomorphism with $\mathcal{G}^{-1}\left(I_{(1)}^{2}\right)=V_{1}+s\left(I_{\mathfrak{e}}\right) R_{o}^{2}$.

Proof. (i) We write $(0)=(0, \ldots, 0) \in \Omega$ for simplicity. Let $\mathbb{G}_{0}$ be the subgroup of $\mathbb{G}$ that consists of the sums $\sum_{t \in \Omega} x_{t}$ with $x_{t} \in \mathbb{Z}\left[\operatorname{Gal}\left(F_{\mathfrak{n}_{t}} / k\right)\right]$ and $x_{(0)} \in I_{(1)}$. Let $\mathcal{F}_{0}$ be the restriction of $\mathcal{F}$ to $\mathbb{G}_{0}$. Since ker $\mathcal{F} \subset \mathbb{G}_{0}$, as one may deduce from Theorem 3.11, we have $\operatorname{ker} \mathcal{F}_{0}=\operatorname{ker} \mathcal{F}$. Moreover, the image $\mathcal{F}_{0}\left(\mathbb{G}_{0}\right)$ is equal to $U_{0}$. Now, we define a homomorphism $\widetilde{\Theta}_{i}: \mathbb{G}_{0} \rightarrow \mathbb{Z} /\left[F_{\mathfrak{p}_{i}} e_{i}: F_{\mathfrak{e}}\right] \mathbb{Z}$ by

$$
\sum_{t \in \Omega} x_{t} \mapsto \operatorname{deg} x_{\langle i\rangle} \bmod \left[F_{\mathfrak{p}_{i}} e_{i}: F_{\mathfrak{e}}\right] .
$$

We see that all the sums $S(t, i, \sigma)$ are in $\operatorname{ker} \widetilde{\Theta}_{i}$. Thus we have $\operatorname{ker} \mathcal{F}_{0}=\operatorname{ker} \mathcal{F} \subset$ ker $\widetilde{\Theta}_{i}$. Consequently, $\widetilde{\Theta}=\left(\widetilde{\Theta}_{1}, \ldots, \widetilde{\Theta}_{s}\right)$ factors through $\mathbb{G}_{0} /$ ker $\mathcal{F}_{0} \simeq U_{0}$ and yields 
the homomorphism $\Theta$. Thus $\Theta$ is well-defined and the surjectivity of $\Theta$ immediately follows from that of $\widetilde{\Theta}$.

By definition of $V_{1}$, it is obvious that $V_{1}+s\left(I_{\mathfrak{e}}\right) R_{0} \subset \operatorname{ker} \Theta$. Let $\sum_{\mathfrak{n} \mid \mathfrak{m}} a_{\mathfrak{n}} \alpha_{\mathfrak{n}} \in$ $\operatorname{ker} \Theta$. Then for each $i=1, \ldots, s$, we have $\sum_{\mathfrak{n} \mid \mathfrak{p}_{i}{ }_{i}} \operatorname{deg} a_{\mathfrak{n}}=d_{i}\left[F_{\mathfrak{p}_{i} e_{i}}: F_{\mathfrak{e}}\right]$ for some $d_{i} \in \mathbb{Z}$. Let $\beta_{i}=\sum_{\sigma \in \operatorname{Gal}\left(F_{\mathbf{p}_{i}} / F_{\mathbf{e}}\right)} \tilde{\sigma}$, where $\tilde{\sigma}$ is an extension of $\sigma$ in $G$. Then we have $\sum_{\mathfrak{n} \mid \mathfrak{p}_{i}^{e_{i}}} a_{\mathfrak{n}} \alpha_{\mathfrak{n}}-d_{i} \beta_{i} \alpha_{\mathfrak{p}_{i} e_{i}} \in R_{0}$. Since $\beta_{i} \alpha_{\mathfrak{p}_{i} e_{i}}=s\left(I_{\mathfrak{e}}\right)\left(1-\bar{\sigma}_{\mathfrak{p}_{i}}\right)=s\left(I_{\mathfrak{e}}\right)\left(1-\mathcal{F}_{\mathfrak{p}_{i}}^{-1}\right) \in$ $s\left(I_{\mathfrak{e}}\right) R_{0}$, we have $\sum_{\mathfrak{n} \mid \mathfrak{m}} a_{\mathfrak{n}} \alpha_{\mathfrak{n}} \in V_{1}+s\left(I_{\mathfrak{e}}\right) R_{0}$. Therefore $\operatorname{ker} \Theta=V_{1}+s\left(I_{\mathfrak{e}}\right) R_{0}$.

(ii) Let $\mathbb{G}_{1}=\operatorname{ker} \tilde{\Theta}$. We define a group homomorphism $\tilde{\mathcal{G}}: \mathbb{G}_{1} \rightarrow I_{(1)}$ by

$$
\tilde{\mathcal{G}}\left(\sum_{t \in \Omega} x_{t}\right)=x_{(0)}+\sum_{i=1}^{s} \frac{\operatorname{deg} x_{\langle i\rangle}}{\left[F_{\mathfrak{p}_{i}}^{e_{i}}: F_{\mathfrak{e}}\right]}\left(1-\left(\mathfrak{p}_{i}, F_{\mathfrak{e}} / k\right)^{-1}\right) \operatorname{res}_{F_{\mathfrak{p}_{i}} / F_{\mathfrak{e}}}\left(x_{\langle i\rangle}\right) .
$$

Since all the $S(t, i, \sigma)$ are contained in $\operatorname{ker} \tilde{\mathcal{G}}, \tilde{\mathcal{G}}$ factors through $\mathbb{G}_{1} / \operatorname{ker} \mathcal{F} \cap \mathbb{G}_{1} \simeq$ $V_{1}+s\left(I_{\mathfrak{e}}\right) R_{0}$ to yield a homomorphism $\mathcal{G}$. Thus $\mathcal{G}$ is well-defined. The surjectivity of $\mathcal{G}$ follows from that of $\tilde{\mathcal{G}}$. It is obvious that $V_{1}+s\left(I_{\mathfrak{e}}\right) R_{0}^{2} \subset \mathcal{G}^{-1}\left(I_{(1)}^{2}\right)$. Let $\sum_{\mathfrak{e} \neq \mathfrak{n} \mid \mathfrak{m}} a_{\mathfrak{n}} \alpha_{\mathfrak{n}}+a_{\mathfrak{e}} s\left(I_{\mathfrak{e}}\right) \in \mathcal{G}^{-1}\left(I_{(1)}^{2}\right)$, that is, $\operatorname{res}_{F / F_{\mathfrak{e}}}\left(a_{\mathfrak{e}}\right) \in I_{(1)}^{2}$. Then we have $s\left(I_{\mathfrak{e}}\right) a_{\mathfrak{e}} \in \operatorname{cor}_{F / F_{\mathfrak{e}}}\left(I_{(1)}^{2}\right)$. Since $\operatorname{cor}_{F / F_{\mathfrak{e}}}\left(I_{(1)}^{2}\right)=\operatorname{cor}_{F / F_{\mathfrak{e}}}\left(\operatorname{res}_{F / F_{\mathfrak{e}}}\left(R_{0}^{2}\right)\right)=s\left(I_{\mathfrak{e}}\right) R_{0}^{2}$, we have $\mathcal{G}^{-1}\left(I_{(1)}^{2}\right) \subset V_{1}+s\left(I_{\mathfrak{e}}\right) R_{0}^{2}$. Therefore $\mathcal{G}^{-1}\left(I_{(1)}^{2}\right)=V_{1}+s\left(I_{\mathfrak{e}}\right) R_{0}^{2}$.

\section{The Stickelberger Ideal}

4.1. Stickelberger ideal. In this subsection, we characterize the Stickelberger ideals following Yin's definition [Y3, Sect. 2,3].

Let $K / k$ be a finite abelian extension with Galois group $G$. We denote by $M_{k}$ the set of all places of $k$ and by $w_{k}$ the number of roots of unity in $k$. Let $T$ be a finite nonempty subset of $M_{k}$ that contains all the places that are ramified in $K$. For a place $v \in M_{k} \backslash T$, let $\mathcal{F}_{v}$ be the Frobenius automorphism associated to $v$ and let $N v$ be the norm of $v$. We define a function for $\operatorname{Re}(s)>1$,

$$
\theta_{K / k, T}(s)=\prod_{v \in M_{k} \backslash T}\left(1-\mathcal{F}_{v}^{-1} N v^{-s}\right)^{-1}
$$

with values in $\mathbb{C}[G]$. Let $\theta_{K, T}=\theta_{K / k, T}(0)$. It is well-known [H2, Thm. 1.1] that $\theta_{K, T} \in \mathbb{Q}[G]$ and $w_{K} \theta_{K, T} \in \mathbb{Z}[G]$. The element $\omega_{K, T}=w_{K} \theta_{K, T}$ is called the Stickelberger element of $K / k$ relative to $T$. It annihilates the group of divisor classes of degree zero of $K$. Let $T_{K}$ be the set of places of $k$ that are ramified in $K$. When $T_{K} \neq \phi$, we let $\theta_{K}=\theta_{K, T_{K}}$. In the cyclotomic function field case, it is known that $\theta_{K_{\mathfrak{f}}}=\omega_{\mathfrak{f}}^{-} \prod_{\mathfrak{p} \mid \mathfrak{f}}\left(1-\bar{\sigma}_{\mathfrak{p}}\right)\left(\left[\underline{Y} 3\right.\right.$ Lemma 4.6]) and $\theta_{K_{\mathfrak{f}}^{+}}=l_{K_{\mathfrak{f}}^{+}}\left(\lambda_{\mathfrak{f}}^{s(J)}\right) /(q-1)$ (Y3, Sect. 3]).

Let $F$ be a finite abelian extension of $k$ with the conductor $\mathfrak{m}=\mathfrak{p}_{1}^{e_{1}} \mathfrak{p}_{2}^{e_{2}} \cdots \mathfrak{p}_{s}^{e_{s}}$. When $F / k$ is a real extension, we define $S_{F}^{-}=0$. When $F / k$ is a non-real extension, we define $S_{F}^{-}$as the $G$-submodule of $\mathbb{Q}[G]$ generated by $\theta_{\mathfrak{f}, F}^{-}=\operatorname{cor}_{F / F_{\mathfrak{f}}} \operatorname{res}_{K_{\mathfrak{f}} / F_{\mathfrak{f}}}\left(\theta_{K_{\mathfrak{f}}}\right)$ with $\mathfrak{f} \mid \mathfrak{m}, \mathfrak{f} \neq \mathfrak{e}$. We also define $S_{F}^{+}$as the $G$-submodule of $\mathbb{Q}[G]$ generated by $\theta_{F}=s(G) /(q-1), \theta_{\mathfrak{p}, F}=\operatorname{cor}_{F / F_{\mathfrak{e}}} \operatorname{res}_{K_{\mathfrak{p}}^{+} / F_{\mathfrak{e}}}\left(\theta_{K_{\mathfrak{p}}^{+}}\right)$for all prime ideals $\mathfrak{p}$ of $\mathbb{A}$ and $\theta_{\mathfrak{f}, F}^{+}=\operatorname{cor}_{F / F_{\mathfrak{f}}^{+}} \operatorname{res}_{K_{\mathfrak{f}}^{+} / F_{\mathfrak{f}}^{+}}\left(\theta_{K_{\mathfrak{f}}^{+}}\right)$with $\mathfrak{f} \mid \mathfrak{m}, \mathfrak{f} \neq \mathfrak{e}$.

Definition 4.1. Let $S_{F}=S_{F}^{+}+S_{F}^{-}$and let $I_{F}=S_{F} \cap R$, called the Stickelberger ideal of the extension $F / k$. We also let $I_{F}^{ \pm}=S_{F}^{ \pm} \cap R$. 
In general, we do not have $I_{F}=I_{F}^{+}+I_{F}^{-}$. Since $\operatorname{deg} \infty=1$, there does not exist a finite extension of $F_{\mathfrak{e}}$ that is abelian over $k$ and in which only $\infty$ is ramified. Thus we have $\theta_{\infty, F}=0$.

\section{Lemma 4.2.}

(i) $\left[S_{F}: I_{F}\right]=\left[S_{F}^{+}: I_{F}^{+}\right]=q-1$.

(ii) $\left[S_{F}^{-}: I_{F}^{-}\right]=(q-1) / \operatorname{gcd}(d, q-1)$, where $d$ is the greatest common divisor of $\left[K_{\mathfrak{f}}: F_{\mathfrak{f}}\right]$ with all $\mathfrak{f} \mid \mathfrak{m}, \mathfrak{f} \neq \mathfrak{e}$.

Proof. Let $\alpha$ be a generator of $\mathbb{F}_{q}^{*}$. Since $(q-1) S_{F} \subset \mathbb{Z}[G], \varphi: S_{F} \rightarrow \mathbb{F}_{q}^{*}$ defined by $\varphi(\theta)=\alpha^{(q-1) a_{1}}$, where $a_{1}$ is the coefficient of 1 in $\theta$, is a well-defined group homomorphism. For any integral ideal $\mathfrak{a}$ of $\mathbb{A}$, relatively prime to $\mathfrak{m}$, we have $((\mathfrak{a}, F / k)-N \mathfrak{a}) \theta \in \mathbb{Z}[G]$ for any $\theta \in S_{F}$. Thus

$$
\varphi((\mathfrak{a}, F / k) \theta)=\varphi(N \mathfrak{a} \theta)=\varphi(\theta)^{N \mathfrak{a}}=\varphi(\theta)=\varphi(\theta)^{(\mathfrak{a}, F / k)},
$$

i.e., $\varphi(\sigma \theta)=\varphi(\theta)^{\sigma}$ for any $\sigma \in G$. Clearly $I_{F}$ is contained in the kernel of $\varphi$. Conversely if $\theta=\sum a_{\sigma} \sigma \in S$ with $\varphi(\theta)=1$, then $(q-1) a_{1} \in(q-1) \mathbb{Z}$. Thus $a_{1} \in \mathbb{Z}$. Since $\varphi$ preserves the $G$-action, $a_{\sigma} \in \mathbb{Z}$ for any $\sigma \in G$. Thus we have $\theta \in I_{F}$ and so $I_{F}=\operatorname{ker}(\varphi)$. Since $\varphi\left(\theta_{F}\right)=\alpha, \varphi$ is a surjective map. Under the same map $\varphi$, we have $\varphi: S_{F}^{+} / I_{F}^{+} \hookrightarrow \mathbb{F}_{q}^{*}$. Since $\theta_{F} \in S_{F}^{+}$, this injection must be a surjection.

By the congruence $Z_{\mathfrak{f}}^{-}(0, \mathfrak{a}) \equiv-1 /(q-1) \bmod \mathbb{Z}$ in $[\mathrm{Y} 3$ Sect. 4$]$, we have that $\theta_{\mathfrak{f}, F}^{-} \equiv-\left[K_{\mathfrak{f}}: F_{\mathfrak{f}}\right] s(G) /(q-1) \bmod \mathbb{Z}[G]$. Thus $\varphi\left(S_{F}^{-}\right)=\left\langle\alpha^{d}\right\rangle$. Since $S_{F}^{-} \cap \operatorname{ker}(\varphi)=$ $I_{F}^{-}$, we get (ii).

Remark 4.3. Since $(q-1) / \delta_{F}$ divides $\left[K_{\mathfrak{f}}: F_{\mathfrak{f}}\right]$ for any $\mathfrak{f} \mid \mathfrak{m}, \mathfrak{f} \neq \mathfrak{e},(q-1) / \delta_{F}$ divides d. Thus $\left[S_{F}^{-}: I_{F}^{-}\right]$is a divisor of $\delta_{F}$.

Corollary 4.4. $S_{F}=I_{F}+\mathbb{Z} \theta_{F}, S_{F}^{+}=I_{F}^{+}+\mathbb{Z} \theta_{F}$.

Proof. Since $I_{F}+\mathbb{Z} \theta_{F} / I_{F} \simeq \mathbb{Z} /(q-1) \mathbb{Z}$, we have $\left[I_{F}+\mathbb{Z} \theta_{F}: I_{F}\right]=q-1$. But $\left[S_{F}: I_{F}\right]=q-1 ;$ so we have $S_{F}=I_{F}+\mathbb{Z} \theta_{F}$. Similarly we have $S_{F}^{+}=I_{F}^{+}+\mathbb{Z} \theta_{F}$.

Lemma 4.5. $e^{+} S_{F}^{+}=S_{F}^{+}$and $e^{-} S_{F}^{-}=S_{F}^{-}$. Thus $S_{F}=S_{F}^{+} \oplus S_{F}^{-}$.

Proof. Since $S_{F}^{+} \subset s\left(J_{F}\right) \mathbb{Q}[G]$, the first equality is obvious. It is known [H2, Sect. 4] that $s(J) \theta_{K_{\mathfrak{f}}}=0$ for any $\mathfrak{f} \neq \mathfrak{e}$. Thus we have $s\left(J_{F}\right) \theta_{\mathfrak{f}, F}^{-}=0$, which implies the second equality.

4.2. Calculation of $\left[R^{-}: I_{F}^{-}\right]$. Let $R^{-}=R \cap e^{-} R$. In this subsection, we assume that $F / k$ is a non-real extension and calculate the index $\left[R^{-}: I_{F}^{-}\right]$.

Lemma 4.6. $S_{F}^{-}=\omega_{F}^{-} e^{-} U$.

Proof. By using the fact that $\theta_{K_{\mathfrak{f}}}=\omega_{\mathfrak{f}}^{-} \prod_{\mathfrak{p} \mid \mathfrak{f}}\left(1-\bar{\sigma}_{\mathfrak{p}}\right)$ and Lemma 2.3. we have

$$
\theta_{\mathfrak{f}, F}^{-}=\omega_{F}^{-} s\left(I_{\mathfrak{f}}\right) \prod_{\mathfrak{p} \mid \mathfrak{f}}\left(1-\bar{\sigma}_{\mathfrak{p}}\right)
$$

for any $\mathfrak{f} \mid \mathfrak{m}, \mathfrak{f} \neq \mathfrak{e}$. Thus we get the result.

We write the index $\left[R^{-}: I_{F}^{-}\right]$as follows:

$$
\left[R^{-}: I_{F}^{-}\right]=\left(R^{-}: e^{-} R\right)\left(e^{-} R: e^{-} U\right)\left(e^{-} U: e^{-} S_{F}^{-}\right)\left(e^{-} S_{F}^{-}: S_{F}^{-}\right)\left(S_{F}^{-}: I_{F}^{-}\right) .
$$


Since $e^{+} R \cap R=s\left(J_{F}\right) R, e^{-} R / R^{-} \simeq e^{+} R / e^{+} R \cap R \simeq s\left(J_{F}\right) R / \delta_{F} s\left(J_{F}\right) R$. Thus we have

$$
\left(R^{-}: e^{-} R\right)=\delta_{F}^{-\left[F^{+}: k\right]},
$$

because $s\left(J_{F}\right) R$ is a free abelian group of rank $\left[F^{+}: k\right]$. By Lemmas 4.5] and 4.6] we have

$$
\begin{aligned}
\left(e^{-} U: e^{-} S_{F}^{-}\right)=\left(e^{-} U: \omega_{F}^{-} e^{-} U\right) & =\operatorname{det}\left(\omega_{F}^{-}\right)=\prod_{\chi \in \widehat{G}^{-}} L_{k}(0, \bar{\chi}) \\
& =h^{-}(F)=\delta^{\left[F^{+}: k\right]-1} h^{-}\left(\mathcal{O}_{F}\right) / Q_{0} .
\end{aligned}
$$

By Lemma 4.5, we have that $e^{-} S_{F}^{-}=S_{F}^{-}$. Thus

$$
\left(e^{-} S_{F}^{-}: S_{F}^{-}\right)=1 \text {. }
$$

By substituting (4.8), (4.4), (4.5) and Lemma 4.2 (ii) into (4.7), we have the following theorem.

Theorem 4.7. $\left[R^{-}: I_{F}^{-}\right]=\frac{q-1}{Q_{0} \delta_{F}(d, q-1)} h^{-}\left(\mathcal{O}_{F}\right)\left(e^{-} R: e^{-} U\right)$.

Remark 4.8. Let $S_{F}^{\prime}$ be the $G$-module generated by the $\theta_{\mathfrak{f}, F}^{-}$with all $\mathfrak{f} \mid \mathfrak{m}$ and $\theta_{F}$. Let $I_{F}^{\prime}=S_{F}^{\prime} \cap R$. Let $\mathcal{A}_{F}=\left\{\vartheta \in \mathbb{Z}[G]: s\left(J_{F}\right) \vartheta \in \mathbb{Z} s(G)\right\}$. When $F / k$ is a non-real extension, we can get the following formula, which is an analogue of Sinnott's (S2. Thm. 2.1]),

$$
\left[\mathcal{A}_{F}: I_{F}^{\prime}\right]=\frac{h^{-}\left(\mathcal{O}_{F}\right)}{Q_{0}}\left(e^{-} R: e^{-} U\right) .
$$

4.3. Calculation of $\left[R^{+}: I_{F}^{+}\right]$. Since $F^{+} / k$ is a real extension, $S_{F^{+}}=S_{F^{+}}^{+}$. Thus

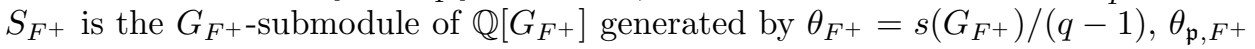
with all prime ideals $\mathfrak{p}$ of $\mathbb{A}$ and $\theta_{\mathfrak{f}, F^{+}}^{+}$with $\mathfrak{f} \mid \mathfrak{m}, \mathfrak{f} \neq \mathfrak{e}$. By the fact that $\theta_{K_{\mathfrak{f}}^{+}}=$ $l_{K_{\mathfrak{f}}^{+}}\left(\lambda_{\mathfrak{f}}^{s(J)}\right) /(q-1)$ and Lemma 2.3] we have $\theta_{\mathfrak{f}, F^{+}}^{+}=l_{F^{+}}\left(\lambda_{\mathfrak{f}, F^{+}}\right) /(q-1)$. Clearly $\theta_{\mathfrak{p}, F^{+}}=l_{F^{+}}\left(N_{K_{\mathfrak{e}} / F_{\mathfrak{e}}}(\xi(\mathbb{A}) / \xi(\mathfrak{p}))\right) /(q-1)$. Let $\bar{P}_{F^{+}}$be the $G_{F^{+}}$-submodule of $\left(F^{+}\right)^{*}$ generated by $N_{K_{\mathfrak{e}} / F_{\mathfrak{e}}}(\xi(\mathbb{A}) / \xi(\mathfrak{p}))$ with all primes $\mathfrak{p}$ and $\lambda_{\mathfrak{f}, F^{+}}$with $\mathfrak{f} \mid \mathfrak{m}, \mathfrak{f} \neq \mathfrak{e}$. Then we have $S_{F^{+}}=l_{F^{+}}\left(\bar{P}_{F^{+}}\right) /(q-1)+\mathbb{Z} \theta_{F^{+}}$.

\section{Lemma 4.9.}

(i) $\left(S_{F^{+}}\right)^{G_{F^{+}}}=\mathbb{Z} \theta_{F^{+}}$.

(ii) $\left(1-e_{1}\right) S_{F^{+}}=\omega_{F^{+}}\left(U_{F^{+}}\right)_{0}$.

Proof. (i) follows from $\left(\bar{P}_{F^{+}}\right)^{G_{F^{+}}}=k \cap \bar{P}_{F^{+}} \subset k_{+}$. Note that $\left(1-e_{1}\right) \theta_{F^{+}}=0$. Then (ii) follows from (3.4).

Lemma 4.10. $\operatorname{cor}_{F / F^{+}}\left(S_{F^{+}}\right)=S_{F}^{+}$and $\operatorname{cor}_{F / F^{+}}\left(I_{F^{+}}\right)=I_{F}^{+}$.

Proof. Since $\operatorname{cor}_{F / F^{+}}\left(\theta_{F^{+}}\right)=\theta_{F}, \operatorname{cor}_{F / F^{+}}\left(\theta_{\mathfrak{p}, F^{+}}\right)=\theta_{\mathfrak{p}, F}$ and $\operatorname{cor}_{F / F^{+}}\left(\theta_{\mathfrak{f}, F^{+}}^{+}\right)=$ $\theta_{\mathfrak{f}, F}^{+}$, we have $\operatorname{cor}_{F / F^{+}}\left(S_{F^{+}}\right) \subset S_{F}^{+}$. Since $\operatorname{cor}_{F / F^{+}}(x) y=\operatorname{cor}_{F / F^{+}}\left(x \operatorname{res}_{F / F^{+}}(y)\right)$ for any $x \in S_{F^{+}}$and $y \in S_{F}^{+}$, the reverse inclusion follows. Similarly, we have $\operatorname{cor}_{F / F^{+}}\left(I_{F^{+}}\right)=I_{F}^{+}$. 
Since $\operatorname{cor}_{F / F^{+}}\left(R_{F^{+}}\right)=s\left(J_{F}\right) R=R^{+}$, we have $\left[R^{+}: I_{F}^{+}\right]=\left[R_{F^{+}}: I_{F^{+}}\right]$by Lemma 4.10, We write the index $\left[R_{F^{+}}: I_{F^{+}}\right]$as follows:

$$
\begin{aligned}
{\left[R_{F^{+}}: I_{F^{+}}\right]=} & \left(R_{F^{+}}: U_{F^{+}}\right)\left(U_{F^{+}}: S_{F^{+}}\right)\left(S_{F^{+}}: I_{F^{+}}\right) \\
= & \left(R_{F^{+}}: U_{F^{+}}\right)\left(s\left(G_{F^{+}}\right) U_{F^{+}}: s\left(G_{F^{+}}\right) S_{F^{+}}\right)\left(\left(U_{F^{+}}\right)_{0}:\left(1-e_{1}\right) S_{F^{+}}\right) \\
& \times\left(\left(1-e_{1}\right) S_{F^{+}}:\left(S_{F^{+}}\right)_{0}\right)\left(S_{F^{+}}: I_{F^{+}}\right) .
\end{aligned}
$$

By Lemma 4.9 (ii), we have

$$
\left(\left(U_{F^{+}}\right)_{0}:\left(1-e_{1}\right) S_{F^{+}}\right)=\operatorname{det}\left(\omega_{F^{+}}\right)=h\left(F^{+}\right) / h(k) .
$$

Since $\left(S_{F^{+}}\right)_{0}=S_{F^{+}} \cap\left(1-e_{1}\right) S_{F^{+}}$and $e_{1} S_{F^{+}} \cap S_{F^{+}}=\left(S_{F^{+}}\right)^{G_{F^{+}}}$,

$$
\left(1-e_{1}\right) S_{F^{+}} /\left(S_{F^{+}}\right)_{0} \simeq e_{1} S_{F^{+}}+S_{F^{+}} / S_{F^{+}} \simeq e_{1} S_{F^{+}} /\left(S_{F^{+}}\right)^{G_{F^{+}}} .
$$

Since $s\left(G_{F^{+}}\right) U_{F^{+}}=\left[F^{+}: F_{\mathfrak{e}}\right] s\left(G_{F^{+}}\right) \mathbb{Z},\left|G_{F^{+}}\right| /\left[F^{+}: F_{\mathfrak{e}}\right]=\left[F_{\mathfrak{e}}: k\right]$, we have (by Lemma 4.9 (i)) that

$$
\begin{aligned}
& \left(\left(1-e_{1}\right) S_{F^{+}}:\left(S_{F^{+}}\right)_{0}\right)\left(s\left(G_{F^{+}}\right) U_{F^{+}}: s\left(G_{F^{+}}\right) S_{F^{+}}\right) \\
& \quad=\left(e_{1} S_{F^{+}}: \mathbb{Z} \theta_{F^{+}}\right)\left(\left[F^{+}: F_{\mathfrak{e}}\right] s\left(G_{F^{+}}\right) \mathbb{Z}: s\left(G_{F^{+}}\right) S_{F^{+}}\right) \\
& \quad=\left(\left[F^{+}: F_{\mathrm{e}}\right] s\left(G_{F^{+}}\right) \mathbb{Z}:\left|G_{F^{+}}\right| \mathbb{Z} \theta_{F^{+}}\right)=\left[F_{\mathfrak{e}}: k\right] /(q-1) .
\end{aligned}
$$

By substituting (4.7), (4.8) and Lemma 4.2 (i) into (4.6), we get the following theorem.

Theorem 4.11. $\left[R^{+}: I_{F}^{+}\right]=\left[R_{F^{+}}: I_{F^{+}}\right]=\frac{h\left(F^{+}\right)}{\left[K_{\mathfrak{e}}: F_{\mathfrak{e}}\right]}\left(R_{F^{+}}: U_{F^{+}}\right)$.

4.4. Calculation of $\left[R: I_{F}\right]$. In this subsection, we calculate the index $\left[R: I_{F}\right]$. Write this index as follows:

$$
\left[R: I_{F}\right]=\left[R: R^{+}+R^{-}\right]\left[R^{+}+R^{-}: I_{F}^{+}+I_{F}^{-}\right]\left[I_{F}: I_{F}^{+}+I_{F}^{-}\right]^{-1} .
$$

Since $\left.\operatorname{ker}\left(e^{+}\right)\right|_{R}=\left.\operatorname{ker}\left(e^{+}\right)\right|_{R^{+}+R^{-}}=R^{-}$and $R^{+}=s\left(J_{F}\right) R$, we have that

$$
\begin{aligned}
{\left[R: R^{+}+R^{-}\right] } & =\left[e^{+} R: e^{+}\left(R^{+}+R^{-}\right)\right]\left[\left.\operatorname{ker}\left(e^{+}\right)\right|_{R}:\left.\operatorname{ker}\left(e^{+}\right)\right|_{R^{+}+R^{-}}\right] \\
& =\left[e^{+} R: R^{+}\right]=\delta_{F}^{\left[F^{+}: k\right]} .
\end{aligned}
$$

Since $R^{+} \cap R^{-}=\{0\}$, we have

$$
\left[R^{+}+R^{-}: I_{F}^{+}+I_{F}^{-}\right]=\left[R^{+}: I_{F}^{+}\right]\left[R^{-}: I_{F}^{-}\right] .
$$

Since $S_{F}^{+} \cap S_{F}^{-}=\{0\}$, we have (by Lemma 4.5) that

$$
\begin{aligned}
{\left[I_{F}: I_{F}^{+}+I_{F}^{-}\right] } & =\left[S_{F}: I_{F}^{+}+I_{F}^{-}\right]\left[S_{F}: I_{F}\right]^{-1} \\
& =\left[S_{F}^{+}: I_{F}^{+}\right]\left[S_{F}^{-}: I_{F}^{-}\right]\left[S_{F}: I_{F}\right]^{-1}=\left[S_{F}^{-}: I_{F}^{-}\right] .
\end{aligned}
$$

By substituting (4.10), (4.11) and (4.12) into (4.9), we get

$$
\left[R: I_{F}\right]=\frac{\delta_{F}^{\left[F^{+}: k\right]}\left[R^{+}: I_{F}^{+}\right]\left[R^{-}: I_{F}^{-}\right]}{\left[S_{F}^{-}: I_{F}^{-}\right]} .
$$

Now we substitute Theorem 4.7 and Theorem 4.11 into (4.13) to obtain the following theorem.

Theorem 4.12. $\left[R: I_{F}\right]=\frac{h(F)}{\left[K_{\mathfrak{e}}: F_{\mathfrak{e}}\right]}\left(e^{-} R: e^{-} U\right)\left(R_{F^{+}}: U_{F^{+}}\right)$. 


\section{Discussion of $(R: U),\left(e^{+} R: e^{+} U\right)$ And $\left(e^{-} R: e^{-} U\right)$}

In this section, we follow Sinnott's arguments in [S2, Sect.5] to obtain some results on the indices $(R: U),\left(e^{+} R: e^{+} U\right)$ and $\left(e^{-} R: e^{-} U\right)$ which appear in our formulas for the index of the group of cyclotomic units and of the Stickelberger ideals.

Recall that $\mathfrak{m}=\mathfrak{p}_{1}^{e_{1}} \mathfrak{p}_{2}^{e_{2}} \cdots \mathfrak{p}_{s}^{e_{s}}$ is the conductor of $F$ and $G=\operatorname{Gal}(F / k)$. Let $\overline{\mathfrak{m}}=\mathfrak{p}_{1} \mathfrak{p}_{2} \cdots \mathfrak{p}_{s}$. For any divisor $\mathfrak{s}$ of $\overline{\mathfrak{m}}$, we denote by $T_{\mathfrak{s}}$ the compositum in $G$ of the inertia groups $T_{\mathfrak{p}}$ as $\mathfrak{p}$ varies through the prime divisors of $\mathfrak{s}$. In particular $T_{\mathfrak{e}}=\{1\}$, $T_{\overline{\mathfrak{m}}}=I_{\mathfrak{e}}$ and in general $T_{\mathfrak{s}}=I_{n(\mathfrak{s})}$, where $n(\mathfrak{s})$ is the largest divisor of $\mathfrak{m}$ coprime to $\mathfrak{s}$. We denote by $U_{\mathfrak{s}}$ the $G$-submodule of $\mathbb{Q}[G]$ generated by the elements

$$
s\left(T_{\mathfrak{r}}\right) \prod_{\mathfrak{p} \mid(\mathfrak{s} / \mathfrak{r})}\left(1-\bar{\sigma}_{\mathfrak{p}}\right)
$$

where $\mathfrak{r}$ varies through the divisors of $\mathfrak{s}$. Hence $U_{\mathfrak{e}}=R$ and $U_{\overline{\mathfrak{m}}}=U$. Following Lemma 5.1 in $\left[\mathbf{S 2}\right.$, one can show that $U_{\mathfrak{s}}$ is a lattice in $\mathbb{Q}[G]$. Let $\mathfrak{p}$ be a prime divisor of $\overline{\mathfrak{m}}$ that does not divide $\mathfrak{s}$. Then we have $U_{\mathfrak{s p}}=U_{\mathfrak{s}}\left(T_{\mathfrak{p}}\right)+\left(1-\bar{\sigma}_{\mathfrak{p}}\right) U_{\mathfrak{s}}$, where $U_{\mathfrak{s}}\left(T_{\mathfrak{p}}\right)$ is the $G$-submodule of $\mathbb{Q}[G]$ generated by the elements

$$
s\left(T_{\mathfrak{r p}}\right) \prod_{\mathfrak{q} \mid(\mathfrak{s} / \mathfrak{r})}\left(1-\bar{\sigma}_{\mathfrak{q}}\right),
$$

where $\mathfrak{r}$ varies through the divisors of $\mathfrak{s}$. Then Lemma 5.1 and the first part of Lemma 5.2 of [S2] hold.

\section{Theorem 5.1.}

(i) The indices $(R: U),\left(e^{+} R: e^{+} U\right)$ and $\left(e^{-} R: e^{-} U\right)$ are integers divisible only by the primes dividing $\left|I_{\mathfrak{e}}\right|$.

(ii) Suppose $I_{\mathfrak{e}}$ is the direct product of the inertia groups $T_{\mathfrak{p}}$ with $\mathfrak{p} \mid \mathfrak{m}$. Then we have $(R: U)=1$.

(iii) If only one prime ramifies in $F$, then $(R: U)=1$, and moreover if $F$ is non-real, then $\left(e^{-} R: e^{-} U\right)=1$ and $\left(e^{+} R: e^{+} U\right)=\delta_{F}^{[G: D]}$. Here $D$ is the decomposition group of the prime that ramifies in $F$. If exactly two primes ramify in $F$, then $(R: U)=1$, and moreover if $F$ is non-real and $J_{F}$ is contained in exactly one inertia group of a ramified prime, then we have $\left(e^{-} R: e^{-} U\right)=1$.

(iv) Suppose that exactly two primes ramify in $F$, say $\mathfrak{p}$ and $\mathfrak{q}$. Let $D_{\mathfrak{p}}$ and $D_{\mathfrak{q}}$ be the decomposition groups of $\mathfrak{p}$ and $\mathfrak{q}$ in $F$, respectively. If $D_{\mathfrak{p}}$ and $D_{\mathfrak{q}}$ are contained in $I_{\mathfrak{e}}$, then we have

$$
\left(e^{+} R: e^{+} U\right)=\frac{\delta_{F}^{\left[G: I_{\mathfrak{e}}\right]} m_{\mathfrak{p}}^{\left[G: J_{F} D_{\mathfrak{p}}\right]} m_{\mathfrak{q}}^{\left[G: J_{F} D_{\mathfrak{q}}\right]}}{\left(m_{\mathfrak{p}}^{\prime} m_{\mathfrak{q}}^{\prime}\right)^{\left[G: I_{\mathfrak{e}}\right]}}
$$

where $m_{\mathfrak{p}}=\left|J_{F} \cap T_{\mathfrak{p}}\right|, m_{\mathfrak{q}}=\left|J_{F} \cap T_{\mathfrak{q}}\right|, m_{\mathfrak{p}}^{\prime}=m_{\mathfrak{p}} /\left(m_{\mathfrak{p}},\left[J_{F} D_{\mathfrak{p}}: J_{F} T_{\mathfrak{p}}\right]\right)$ and $m_{\mathfrak{q}}^{\prime}=m_{\mathfrak{q}} /\left(m_{\mathfrak{q}},\left[J_{F} D_{\mathfrak{q}}: J_{F} T_{\mathfrak{q}}\right]\right)$.

Proof. (i) and (ii) follow from exactly the same process of [S2, Prop. 5.1, Thm. 5.4].

(iii) The indices $(R: U)$ and $\left(e^{-} R: e^{-} U\right)$ are calculated as [S2, Prop. 5.2]. When exactly two primes ramify in $F$, we can calculate $\left(e^{-} R: e^{-} U\right)$ only for the case that $J_{F}$ is contained in either inertia group. This is because we only have the first part (i.e., $H^{1}\left(H, R^{K}\right)=0$ ) of [S2, Lemma 5.2] in our case. 
Since $\left(e^{+} R\right)^{T_{\mathfrak{p}}}=\left(1 /\left|J_{F} \cap T_{\mathfrak{p}}\right|\right) e^{+} s\left(T_{\mathfrak{p}}\right) R=\left(1 / \delta_{F}\right) s\left(J_{F} T_{\mathfrak{p}}\right) R$, we have

$$
\left(e^{+} R: e^{+} U_{\mathfrak{p}}\right)=\left|s\left(J_{F} T_{\mathfrak{p}}\right) R /\left(\left|J_{F} \cap T_{\mathfrak{p}}\right| s\left(J_{F} T_{\mathfrak{p}}\right) R+\left(1-\mathcal{F}_{\mathfrak{p}}^{-1}\right) s\left(J_{F} T_{\mathfrak{p}}\right) R\right)\right| .
$$

Since the group on the right-hand side of (5.1) is isomorphic to

$$
\left(\mathbb{Z} /\left|J_{F} \cap T_{\mathfrak{p}}\right| \mathbb{Z}\right)\left[G / J_{F} D_{\mathfrak{p}}\right]
$$

we have

$$
\left(e^{+} R: e^{+} U_{\mathfrak{p}}\right)=\left|J_{F} \cap T_{\mathfrak{p}}\right|^{\left[G: J_{F} D_{\mathfrak{p}}\right]} .
$$

(iv) Let $e_{\mathfrak{p}}=s\left(T_{\mathfrak{p}}\right) /\left|T_{\mathfrak{p}}\right|$. Following the proof of [S2, Thm.5.1], we see that

$$
\left(e^{+} U_{\mathfrak{p}}: e^{+} U_{\mathfrak{p q}}\right)=\left|B_{1} /\left(1-\mathcal{F}_{\mathfrak{q}}^{-1}\right) B_{1}\right| \times\left|B_{2} /\left(1-\mathcal{F}_{\mathfrak{q}}^{-1}\right) B_{2}\right|,
$$

where $B_{1}=\left(e^{+} U_{\mathfrak{p}}\right)^{T_{\mathfrak{p q}}} / e^{+} s\left(T_{\mathfrak{p q}}\right) R=\left(e^{+} U_{\mathfrak{p}}\right)^{I_{\mathfrak{e}}} / s\left(I_{\mathfrak{e}}\right) R$ and

$$
B_{2}=\left(1-e_{\mathfrak{p}}\right)\left(e^{+} R\right)^{T_{\mathfrak{q}}} /\left(1-e_{\mathfrak{p}}\right) e^{+} s\left(T_{\mathfrak{q}}\right) R \simeq\left(e^{+} R\right)^{T_{\mathfrak{q}}} /\left(e^{+} s\left(T_{\mathfrak{q}}\right) R+\left(1 / \delta_{F}\right) s\left(I_{\mathfrak{e}}\right) R\right) .
$$

Since $\left(e^{+} R\right)^{T_{\mathfrak{p}}}=\left(1 / m_{\mathfrak{p}}\right) e^{+} s\left(T_{\mathfrak{p}}\right) R=\left(1 / \delta_{F}\right) s\left(J_{F} T_{\mathfrak{p}}\right) R$, we have

$$
\left(e^{+} U_{\mathfrak{p}}\right)^{T_{\mathfrak{p}}}=\left(1 / \delta_{F}\right)\left(m_{\mathfrak{p}} s\left(J_{F} T_{\mathfrak{p}}\right) R+\left(1-\mathcal{F}_{\mathfrak{p}}^{-1}\right) s\left(J_{F} T_{\mathfrak{p}}\right) R\right) .
$$

It is easy to see that $\left(m_{\mathfrak{p}} s\left(J_{F} T_{\mathfrak{p}}\right) R+\left(1-\mathcal{F}_{\mathfrak{p}}^{-1}\right) s\left(J_{F} T_{\mathfrak{p}}\right) R\right)^{J_{F} D_{\mathfrak{p}}}=m_{\mathfrak{p}}^{\prime} s\left(J_{F} D_{\mathfrak{p}}\right) R$. Thus $\left(e^{+} U_{\mathfrak{p}}\right)^{I_{\mathfrak{e}}}=\left(1 / \delta_{F}\right)\left(m_{\mathfrak{p}}^{\prime} s\left(J_{F} D_{\mathfrak{p}}\right) R\right)^{I_{\mathfrak{e}}}=\left(1 / \delta_{F}\right) m_{\mathfrak{p}}^{\prime} s\left(I_{\mathfrak{e}}\right) R$. Since $D_{\mathfrak{q}}$ is contained in $I_{\mathfrak{e}},\left(1-\mathcal{F}_{\mathfrak{q}}^{-1}\right) B_{1}=0$. Therefore we have

$$
\left|B_{1} /\left(1-\mathcal{F}_{\mathfrak{q}}^{-1}\right) B_{1}\right|=\left|B_{1}\right|=\left(\delta_{F} / m_{\mathfrak{p}}^{\prime}\right)^{\left[G: I_{\mathfrak{e}}\right]} .
$$

Since $\left(e^{+} R\right)^{T_{\mathfrak{q}}}=\left(1 / m_{\mathfrak{q}}\right) e^{+} s\left(T_{\mathfrak{q}}\right) R=\left(1 / \delta_{F}\right) s\left(J_{F} T_{\mathfrak{q}}\right) R$, we have

$$
B_{2} \simeq s\left(J_{F} T_{\mathfrak{q}}\right) R /\left(m_{\mathfrak{q}} s\left(J_{F} T_{\mathfrak{q}}\right) R+s\left(I_{\mathfrak{e}}\right) R\right) \simeq\left(\mathbb{Z} / m_{\mathfrak{q}} \mathbb{Z}\right)\left[G / J_{F} T_{\mathfrak{q}}\right] /\left(s\left(I_{\mathfrak{e}} / J_{F} T_{\mathfrak{q}}\right)\right),
$$

and so

$$
\frac{B_{2}}{\left(1-\mathcal{F}_{\mathfrak{q}}^{-1}\right) B_{2}} \simeq \frac{\left(\mathbb{Z} / m_{\mathfrak{q}} \mathbb{Z}\right)\left[G / J_{F} D_{\mathfrak{q}}\right]}{s\left(I_{\mathfrak{e}} / J_{F} D_{\mathfrak{q}}\right)\left[J_{F} D_{\mathfrak{q}}: J_{F} T_{\mathfrak{q}}\right]\left(\mathbb{Z} / m_{\mathfrak{q}} \mathbb{Z}\right)\left[G / J_{F} D_{\mathfrak{q}}\right]}
$$

Since $\left|\left[J_{F} D_{\mathfrak{q}}: J_{F} T_{\mathfrak{q}}\right]\left(\mathbb{Z} / m_{\mathfrak{q}} \mathbb{Z}\right)\right|=m_{\mathfrak{q}}^{\prime}$, we get

$$
\left|B_{2} /\left(1-\mathcal{F}_{\mathfrak{q}}^{-1}\right) B_{2}\right|=m_{\mathfrak{q}}^{\left[G: J_{F} D_{\mathfrak{q}}\right]} / m_{\mathfrak{q}}^{\prime\left[G: I_{\mathfrak{e}}\right]} .
$$

Finally, by (5.2) and by substituting (5.4) and (5.5) into (15.3), we have

$$
\left(e^{+} R: e^{+} U_{\mathfrak{p q}}\right)=\left(e^{+} R: e^{+} U_{\mathfrak{p}}\right)\left(e^{+} U_{\mathfrak{p}}: e^{+} U_{\mathfrak{p q}}\right)=\frac{\delta_{F}^{\left[G: I_{\mathfrak{e}}\right]} m_{\mathfrak{p}}^{\left[G: J_{F} D_{\mathfrak{p}}\right]} m_{\mathfrak{q}}^{\left[G: J_{F} D_{\mathfrak{q}}\right]}}{\left(m_{\mathfrak{p}}^{\prime} m_{\mathfrak{q}}^{\prime}\right)^{\left[G: I_{\mathfrak{e}}\right]}}
$$

which completes the proof.

\section{ACKNOWLEDGMENTS}

The authors would like to express their sincere gratitude to Professors Linsheng Yin and Hassan Oukhaba for their interest and helpful suggestions. They thank also the referee for his careful reading of the manuscript and suggestions on writing of the paper. 


\section{REFERENCES}

[B] J.-R. Belliard, Sur la structure galoisienne des unités circulaires dans les $\mathbf{Z}_{p}$-extensions. J. Number Theory 69 (1998), no. 1, 16-49. MR 98k:11164

[BO] J.-R. Belliard and H. Oukhaba, Sur la torsion de la distribution ordinaire universelle attachée à un corps de nombres. Manuscripta Math. 106 (2001), no. 1, 117-130. MR 2002f:11163

[GR] S. Galovich and M. Rosen, Units and class groups in cyclotomic function fields. J. Number Theory 14 (1982), 156-184. MR 84b:12008

[Hr] F. Harrop, Circular units of function fields. Trans. Amer. Math. Soc. 341 (1994), 405-421. MR 94c:11106

[H1] D. Hayes, Elliptic units in function fields. Number theory related to Fermat's last theorem (Cambridge, Mass. 1981), 321-340; Progress in Mathematics 26, Birkhäuser, Boston, 1982. MR 84f:12005

[H2] D. Hayes, Stickelberger elements in function fields. Compositio Math. 55 (1985), 209-239. MR 87d:11091

[O] H. Oukhaba, Index formulas for ramified elliptic units, To appear in Compositio Math.

[S1] W. Sinnott, On the Stickelberger ideal and the circular units of a cyclotomic field. Ann. of Math. (2) 108 (1978), 107-134. MR 58:5585

[S2] W. Sinnott, On the Stickelberger ideal and the circular units of an abelian field. Invent. Math. 62 (1980/81), 181-234. MR 82i:12004

[T] J. Tate, Les conjectures de Stark sur les fonctions L d'Artin en $s=0$. Progress in Mathematics 47, Birkhäuser, Boston, 1984. MR 86e:11112

[Y1] L. Yin, Index-class number formulas over global function fields. Compositio Math. 109 (1997), 49-66. MR 98h:11151

[Y2] L. Yin, Stickelberger ideals and relative class numbers in function fields. J. Number Theory 81 (2000), 162-169. MR 2001d:11114

[Y3] L. Yin, Stickelberger ideals and divisor class numbers. Math. Z. 239 (2002), no. 3, 425-440.

Department of Mathematics, KAIST Daejon, 305-701, Korea

E-mail address: jaehyun@mathx.kaist.ac.kr

Department of Mathematics, KAIST Daejon, 305-701, Korea

E-mail address: shbae@math.kaist.ac.kr

Department of Mathematics, KAist Daejon, 305-701, Korea

E-mail address: hyjung@mathx.kaist.ac.kr 